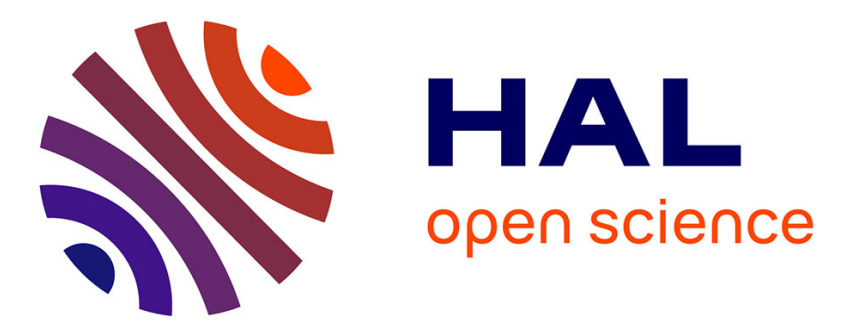

\title{
Place du Bayacien dans la structuration du Gravettien
}

Damien Pesesse

\section{To cite this version:}

Damien Pesesse. Place du Bayacien dans la structuration du Gravettien. Gallia Préhistoire - Préhistoire de la France dans son contexte européen, 2008, 50, pp.23-44. 10.3406/galip.2008.2457 . halshs00409183

\section{HAL Id: halshs-00409183 \\ https://shs.hal.science/halshs-00409183}

Submitted on 2 Jan 2020

HAL is a multi-disciplinary open access archive for the deposit and dissemination of scientific research documents, whether they are published or not. The documents may come from teaching and research institutions in France or abroad, or from public or private research centers.
L'archive ouverte pluridisciplinaire HAL, est destinée au dépôt et à la diffusion de documents scientifiques de niveau recherche, publiés ou non, émanant des établissements d'enseignement et de recherche français ou étrangers, des laboratoires publics ou privés.

\section{(1) (1) $\$$}

Distributed under a Creative Commons Attribution - NonCommercial - NoDerivatives| 4.0 


\title{
PLACE DU BAYACIEN DANS LA STRUCTURATION DU GRAVETTIEN
}

\author{
Damien PESESSE*
}

Mots-clés. Fléchette, système technique, évolution culturelle.

Résumé. L'émergence de nouvelles traditions sur l'ensemble de l'Europe autour de 29000 B.P. a profondément modifié le paysage culturel. Cet épisode crucial de l'histoire des populations paléolithiques demeure pourtant très peu documenté. En France, le Bayacien, reconnu à la Gravette (Bayac, Dordogne), est interprété comme la plus ancienne manifestation du Gravettien. Le Bayacien est caractérisé par la prédominance des fléchettes. L'originalité et la cohérence de cette industrie sont évaluées par une approche globale du système technique lithique. Cette étude confirme l'originalité du Bayacien au vu des productions aurignaciennes et gravettiennes. Ce premier Gravettien n'est pas un Gravettien stricto sensu, mais les valeurs gravettiennes se dessinent déjà sous la forme bayacienne.

Key-words. Flechette, technical system, cultural evolution.

Abstract. Around 29,000 B.P., the emergence of new traditions profoundly modified the European cultural landscape. Meanwhile, the processes of this crucial episode in the history of Palaeolithic populations remain poorly known. In France, the Bayacian, recognized at la Gravette (Bayac, Dordogne) and characterized by a predominance of flechettes, is interpreted as the oldest manifestation of the Gravettian. The technological study presented here confirms the originality and the specificity of this industry relative to Gravettian and Aurignacian industries. Even if no Gravette points have been found in the Bayacian, the emergence of a highly invested projectile element during this period can be interpreted as the first manifestation of Gravettian values. From this perspective, this first Gravettian is not a Gravettian stricto sensu, but shows the first signs of the emergence of this culture.

Translation: Magen O'FARRELL

Schlüsselwörter. Wurfpfeil, technisches System, kulturelle Evolution.

Zusammenfassung. Die europaweite Herausbildung neuer Traditionen um 29000 B.P. hat die Kulturlandschaft tief greifend verändert. Dennoch ist dieser bedeutende Abschnitt in der Geschichte der paläolithischen Völker immer noch sehr spärlich dokumentiert. In Frankreich wird das in la Gravette (Bayac, Dordogne) nachgewiesene Bayacien als die älteste Äußerung des Gravettien interpretiert. Das Bayacien charakterisiert sich durch das Vorherrschen von Wurfpfeilen. Die Originalität und die Kohärenz dieser Industrie werden in diesem Artikel untersucht und anhand eine globalen Betrachtung der lithischen Industrie überprüft. Die vorliegende Studie bestätigt die Originalität des Bayacien hinsichtlich der Produktionen des Aurignacien und des Gravettien. Dieses frühe Gravettien ist kein Gravettien stricto sensu, doch die Werte des Gravettien zeichnen in der Gestalt des Bayacien bereits ab.

Übersetzung: Isa ODENHARDT-DONVEZ

Le gisement de la Gravette (commune de Bayac, Dordogne) a livré une séquence de cinq horizons archéologiques. La stratigraphie débute par un Aurignacien récent et se termine par trois niveaux se rapportant au Gravettien ancien. Entre ces deux entités, F. Lacorre mit au jour en 1930 un horizon qui ne partage, d'après lui, ni les caractères de l'Auri- gnacien ni du Gravettien (Lacorre, 1933a et 1933b). Cette spécificité technique alliée à sa position chronostratigraphique conduisit F. Lacorre à l'individualiser sous l'appellation «Bayacien » (Lacorre, 1960). Nul ensemble strictement identique ne fut découvert depuis. Le Bayacien compte ainsi parmi les rares « hapax archéologiques » du Paléolithique.

* LAMPEA, Université de Provence, UMR 6636 du CNRS, MCC, IRD, Maison méditerranéenne des sciences de l'homme, 5 rue du Château-del'Horloge, BP 647, F-13094 Aix-en-Provence Cedex 2. Courriel : pesesse@mmsh.univ-aix.fr 
Depuis la publication de l'importante monographie en 1960, seul H. Delporte dans le cadre des fouilles du musée des Antiquités nationales réexamina cette série lithique (Delporte, 1972). Cet auteur questionne la cohérence de l'ensemble, sans bénéficier alors des outils méthodologiques adéquats pour la vérifier.

Les raisons de cette singularité et la pertinence de cette distinction demandent donc à être abordées. L'intégrité de l'assemblage et l'évaluation de sa spécificité tiennent alors une place centrale. En l'absence de documentation provenant des fouilles de F. Lacorre, l'approche technologique constitue un moyen privilégié pour tester la cohérence de l'ensemble. Plusieurs conditions sont requises a priori pour statuer sur ce point, notamment la cohérence des systèmes techniques, le degré de fait des schémas opératoires et leur éventuelle convergence avec les procédés documentés dans le gisement.

La caractérisation du Bayacien s'avère nécessaire car cette industrie tient une place notable dans la sériation du Gravettien. Ce niveau est en effet considéré comme l'une des premières manifestations du Gravettien en France (Peyrony, 1933; Sonneville-Bordes, 1960; Otte, 1981 et 1990; Bosselin, Djindjian, 1994; Djindjian, Bosselin, 1994; Rigaud, 1998; Otte, Noiret, 2004).

\section{LA GRAVETTE}

\section{LE GISEMENT}

Le gisement est situé à Bayac (Dordogne) en bordure de la Couze, à 4,50 km de la confluence avec la Dordogne. Cette vallée relativement étroite témoigne d'une fréquentation paléolithique importante, d'après le nombre de stations, toutes situées sur la rive droite de la Couze et orientées vers le sud. Parmi ces gisements, le Roc de Combe-Capelle à Saint-Avit-Sénieur (Dordogne) est également attribuable au Gravettien (Peyrony, 1943).

La Gravette se situe au pied d'une falaise dont la morphologie à hauteur du gisement n'est plus discernable, compte tenu de l'effondrement ancien de la paroi et de son utilisation comme carrière au début du siècle. Un ressaut rocheux pouvait donc exister à l'origine.

L'invention du gisement revient à Mgr Chastaing et remonte au printemps de l'année 1880. Les premières «fouilles » furent menées par Messieurs Tabanou, instituteur à Monsac, et Coste, bijoutier à Issigeac. Ces collections furent vendues et partagées entre différents musées, dont le musée d'Aquitaine, le musée de Périgueux et le musée du Latran à Rome; certaines arrivèrent aux États-Unis. Par la suite, l'abbé Landesque reprit les fouilles, dont le fruit fut vendu par sa famille. De nombreux prospecteurs leur succédèrent, dont Hardy, Délugin, Féaux et le professeur Testut, jusqu'à ce que le gisement soit de nouveau délaissé. Des premières fouilles survécut l'idée d'un unique niveau de Gravettien livrant parmi les premières pointes de la Gravette (Lacorre, 1960).

Le 14 juillet 1930, " par un soleil de plomb », F. Lacorre aborda la station, devancé par son épouse qui entama le gisement, munie d'une pioche (Lacorre, 1960). La fouille dura jusqu'à la Seconde Guerre mondiale, reprenant par la suite, dès 1945 et jusqu'en 1949. Une dernière campagne eut lieu durant les années 1953 et 1954.

F. Lacorre mit en évidence une stratigraphie développée sur $5 \mathrm{~m}$ d'épaisseur. Le premier niveau, aurignacien, se situe en contact direct avec le substrat. Le Bayacien et les niveaux gravettiens sont compris dans les trois premiers mètres, contenus dans une matrice caillouteuse calcaire. La séquence est clôturée par un niveau d'effondrement de gros blocs qui scelle les niveaux archéologiques sur $2 \mathrm{~m}$ d'épaisseur. Aucune occupation paléolithique postérieure n’est enregistrée.

\section{LE NIVEAU BAYAGIEN}

Le niveau bayacien fut découvert par F. Lacorre lors de sa première campagne de fouille (Lacorre, 1933a), les occupations gravettiennes sus-jacentes étant progressivement décalées vers l'ouest. La deuxième campagne lui permit de vérifier la position relative de cette industrie à fléchettes (Lacorre, 1933b). Lacorre réalisa trois tranchées, distantes respectivement de 5,90 m et 2,25 m. Des ensembles attribués au Bayacien furent retrouvés dans chacune des trois tranchées. Sa répartition varie latéralement avec une plus forte concentration du mobilier archéologique dans la zone est du gisement, au niveau du foyer (tranchée 1). Dans ce secteur, la fin de la première couche gravettienne est directement sus-jacente au Bayacien, alors qu'elle en est séparée par un niveau stérile dans le secteur central (Lacorre, 1960). Sur une surface de $28 \mathrm{~m}^{2}$, plus de 200 fléchettes furent découvertes. Ces pièces, dénommées également " pointes de Bayac ", firent l'objet de plusieurs définitions mettant l'accent sur la morphologie foliacée, sublosangique, la minceur de la pièce, le caractère court et périphérique de la retouche (Brézillon, 1968; Demars, Laurent, 1989). 


\section{LA COLLECTION}

La série bayacienne, comme l'ensemble de la collection Lacorre, a été donnée à deux musées, le musée d'Archéologie nationale, qui reçut également le gisement, et le musée-forum d'Aurignac. La répartition de la série ne fut pas égale entre ces institutions. La collection bayacienne de Saint-Germain-en-Laye représente presque les deux tiers de la série et plus de $80 \%$ des fléchettes (tabl. I). Malgré cette différence, ces deux collections se répondent parfaitement. Avec un effectif de 343 pièces (218 au musée d'Archéologie nationale et 125 au musée-forum d'Aurignac), cette série s'avère quantitativement restreinte. Les comportements techniques peuvent toutefois être envisagés avec une certaine précision, compte tenu du nombre important de produits finis (179 armatures) et de la lisibilité du schéma de production de fléchettes. La description réalisée par F. Lacorre, par sa précision et sa justesse, peut d'ailleurs figurer en bonne place parmi les textes annonciateurs des études technologiques (Lacorre, 1933a).

\section{PRÉSENTATION DE L'INDUSTRIE}

L'industrie bayacienne est structurée à partir d'une armature axiale sublosangique: la fléchette. L'obtention de la « pointe de Bayac » constitue l'aspect le plus marquant de l'assemblage archéologique, rassemblant 73,5\% des outils, supports et nucléus (tabl. I). Aucun autre type d'armature n'est représenté; la pointe de la Gravette, en particulier, est absente. La prépondérance de cette armature confère à cette industrie un caractère extrêmement léger et élégant.

Deux autres intentions techniques sont documentées; l'une correspond à une recherche de petites lames (uniquement représentée par six individus), l'autre à un débitage laminaire massif à finalité domestique (18\% de la série). Ces objectifs ne présentent a priori aucune solution de continuité entre eux et pourront donc être abordés séparément.

Les matériaux siliceux prélevés renvoient à une exploitation essentiellement locale du territoire: silex maestrichtien du bergeracois, silex sénonien issu des bancs calcaires environnants, silex tertiaires et de rares pièces en silex turonien du Fumélois. Les restes fauniques conservés se rapportent au Renne, au Cheval et au Mammouth (Lacorre, 1960). Les témoins de l'exploitation des matières dures animales se restreignent à des fragments d'objets appointés, des hémi-côtes obtenues par bipartition dont certaines pourraient correspondre à des lissoirs, des déchets d'élagage sur andouillers de renne (Goutas, 2004).
Tabl. I - Inventaire de la série bayacienne.

\begin{tabular}{|c|c|c|c|c|}
\hline & \multirow{3}{*}{$\begin{array}{c}\text { Saint-Germain } \\
145\end{array}$} & \multirow{3}{*}{\begin{tabular}{|c|} 
Aurignac \\
34
\end{tabular}} & \multirow{3}{*}{$\begin{array}{l}\text { Total } \\
179\end{array}$} \\
\hline & & & & \\
\hline \multirow{4}{*}{ Fléchette } & fléchette & & & \\
\hline & produit & - & 27 & 27 \\
\hline & sous-produit & - & 39 & 39 \\
\hline & nucléus & 6 & 1 & 7 \\
\hline \multirow{3}{*}{ Petite lame } & produit & 2 & 1 & 2 \\
\hline & sous-produit & - & 1 & 1 \\
\hline & nucléus & 2 & - & 3 \\
\hline \multirow{3}{*}{ Lame } & produit & 42 & 15 & 57 \\
\hline & sous-produit & 2 & - & 2 \\
\hline & nucléus & 3 & - & 3 \\
\hline \multicolumn{2}{|c|}{ Nucléus indéterminé } & 3 & - & 3 \\
\hline \multicolumn{2}{|l|}{ Éclat } & 12 & - & 12 \\
\hline \multicolumn{2}{|c|}{ Éclat lamellaire } & - & 2 & 2 \\
\hline \multicolumn{2}{|c|}{ Chute de burin } & 1 & 5 & 6 \\
\hline & Total & 218 & 125 & 343 \\
\hline
\end{tabular}

\section{PRODUCTION ET TRANSFORMATION DES FLÉCHETTES}

\section{LA PRODUCTION}

Les fléchettes partagent un ensemble de critères morphométriques corrélables à une certaine standardisation des chaînes opératoires, ce qui sous-entend un investissement technique important. Le produit recherché est peu épais, large par rapport à sa longueur, de morphologie sublosangique, à extrémité distale aiguë. Le débitage est mené à partir d'un plan de frappe principal, sur un volume entièrement mis en forme (fig. 1). Le dos est aménagé par une série d'enlèvements, globalement transversaux; le plan de frappe, d'angulation ouverte, reste lisse. La base et les flancs sont aménagés à partir d'un plan de frappe opposé décalé. La table est rectiligne, large, très peu cintrée, se resserrant dans la partie distale. La structure volumétrique des six nucléus témoigne d'une réelle homogénéité.

Les premiers produits sont relativement élancés et s'apparentent à de petites lames, car ils bénéficient de conditions volumétriques nécessaires à l'initialisation du débitage, la mise en place de nervures guides. Le rôle de ces pièces dans la configuration du volume s'observe notamment au niveau de l'extrémité distale par une légère rupture de profil ou par une épaisseur plus marquée. Avec la réduction du volume, les convexités vont s'amoindrir sans être restaurées de manière aussi nette. Les caractéristiques 

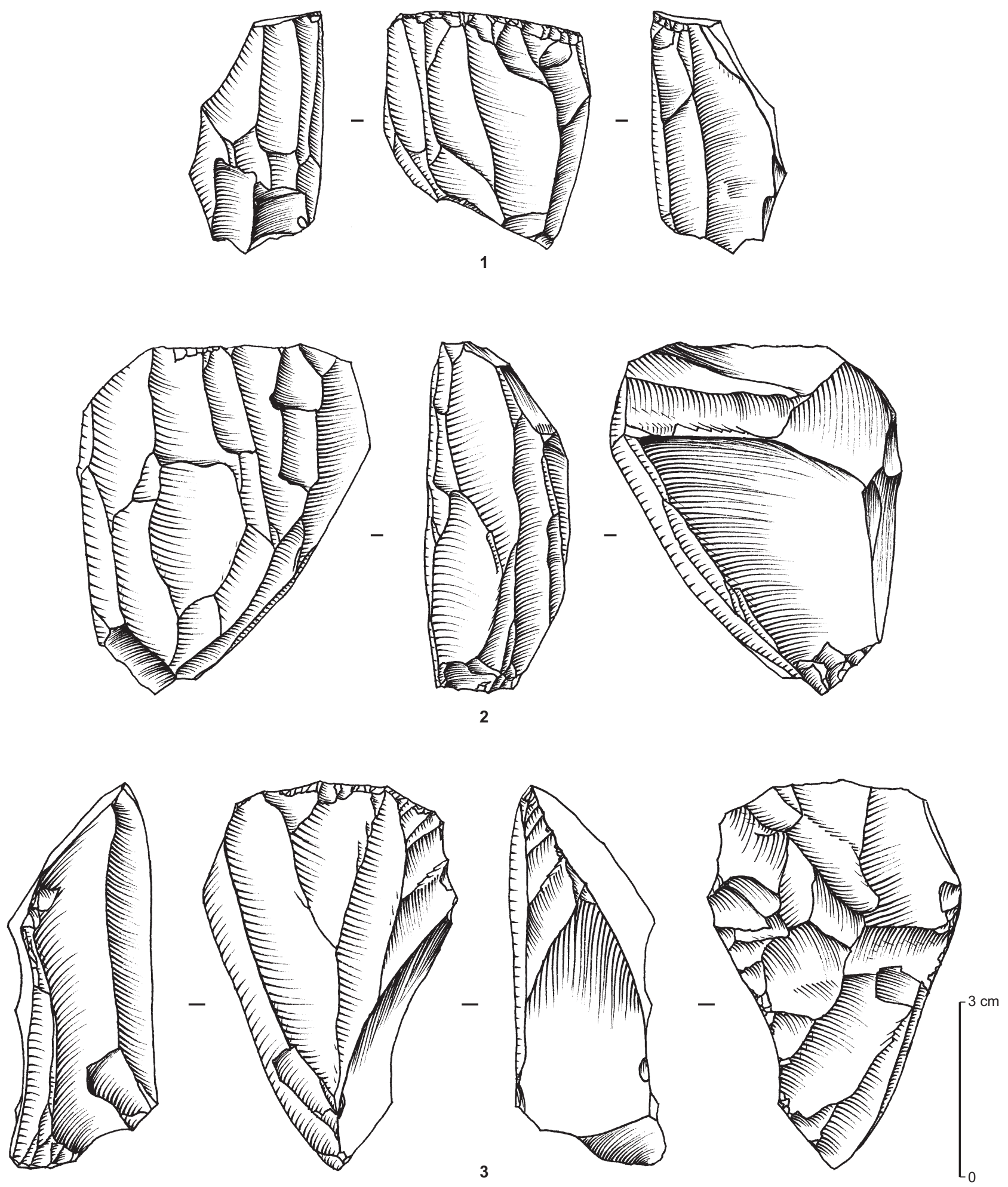

Fig. 1 - Nucléus à fléchettes (dessin: D. Pesesse). 
des produits se voient ainsi modifiées pour atteindre pleinement l'objectif. Le caractère peu marqué du cintre garantit l'étalement latéral de l'onde de choc qui assure la largeur requise du support. La présence d'un dièdre en partie basale, liée notamment à la convergence des enlèvements, canalise l'onde de choc sur toute la longueur de la table et rend naturellement acuminée l'extrémité des produits.

La présence d'un plan de frappe à la base de la table joue un rôle important dans le déroulement du débitage; il infère en effet sur de nombreux paramètres dont l'incidence sur la morphologie des produits apparaît immédiatement. Les enlèvements investissent uniquement les flancs du nucléus, délimitant latéralement la table sans déborder et assurant la convergence des enlèvements extraits sur une table large. Les flancs possèdent ainsi une morphologie convexe, un galbe caractéristique. Leur faible débordement sur la table se traduit par le petit nombre de produits latéraux à enlèvement opposé latéralisé ( $\mathrm{N}=4$, voir fig. 2, $n^{\circ} 8$; fig. $6, n^{\text {os }} 6$ et 9 ; fig. $7, n^{\circ} 8$ ).

À titre d'hypothèse, l'utilisation d'un plan de frappe opposé peut s'expliquer par une volonté de limiter l'investissement des flancs à partir du plan de frappe principal, ce qui induirait une torsion des supports situés en bord de table. Or, les produits latéraux sont également utilisés pour la confection de fléchettes et peu d'entre elles présentent une torsion marquée en partie proximale. L'ensemble des produits extraits depuis le plan de frappe principal est donc recherché; les plus calibrés sont transformés, mais également ceux destinés à reprendre ou réorienter une nervure, rabaisser une convexité trop forte ou éliminer une irrégularité. Un enchaînement subtil de supports à fonctions différenciées apparaît ainsi, témoignant du soin accordé constamment à l'entretien des paramètres optimaux de production sans impliquer une phase autonome de réaménagement. Seules les interventions depuis la base du volume peuvent être considérées comme des éléments de rupture. La fréquence de la mobilisation du plan de frappe opposé ne peut malheureusement être appréciée.

Tout l'art réside donc dans la manière de faire varier en différents points l'étalement latéral de l'onde de choc. En partie proximale, depuis la zone de contact du percuteur ${ }^{1}$, l'onde de choc s'évase, s'élargit en partie mésiale, puis se resserre en partie distale. Le produit ainsi obtenu est déjà une fléchette, et non une lame stricto sensu. Il présente un

1. L'utilisation de la percussion organique prédomine sur la pierre tendre, représentée par quelques exemplaires tout à fait caractéristiques d'après les caractères décrits par J. Pelegrin (2000). équilibre naturel, une nervure centrale, ou plusieurs convergentes, et des bords dont la délinéation est tributaire de sa fonction et de sa position sur la table, mais de morphologie fréquemment biconvexe.

\section{VARIANTE}

Une manière de faire se démarque du schéma précédent. Dans ce cas, la production est réalisée de manière unipolaire à partir de volumes resserrés. La gestion globale du volume est assurée par un investissement plus important des flancs, par des supports de recadrage (fig. 3, n ${ }^{\text {os }} 3$ et 4 ). Les produits extraits au centre d'une table cintrée ne décrivent donc pas l'étalement latéral observé pour le premier schéma. Il s'agit bien de petites lames à bords subparallèles dont l'extrémité est rendue aiguë par la convergence des enlèvements. Dans cette série, les rares néocrêtes latérales et supports de recadrage, modalités liées au débordement vers les flancs, appartiennent à ce schéma (fig. 3, no 1). Ces pièces présentent également une pétrographie commune. Le nucléus, les produits et sous-produits relevant de ce schéma sont tous en silex tertiaire, excepté un nucléus en Bergeracois (fig. 3, $n^{\circ} 5$ ).

\section{LES FLÉCHETTES}

La transformation concourt à affiner les caractéristiques définies par la production, en renforçant une extrémité, réajustant la délinéation d'un bord, par une retouche très limitée en extension et en intensité. Les variations morphologiques du support expliquent en partie la diversité de l'aménagement reconnue par H. Delporte (cette étude porte sur 119 pièces, Delporte, 1972). D’après cet auteur, aucun paramètre-direction, intensité, répartition - ne serait stable. Sur quelques pièces, $H$. Delporte constate une tendance à la latéralisation et une retouche parfois abrupte. Une certaine inadéquation apparaît donc entre la norme de production, rigide, et la norme de transformation, souple, conduisant à une relative hétérogénéité. Dans quelques cas, le produit est même transformé a contrario de ses caractéristiques.

Ainsi, la symétrie et le caractère acuminé du support sont parfois modifiés en déplaçant latéralement l'apex et/ou la base par une fine troncature distale oblique droite directe (fig. 4). Dans ce cas, la partie aiguë est toujours déplacée vers la droite. Cette modification s'accompagne d'une retouche directe complète du bord droit, lui conférant une délinéation globalement droite ou légèrement convexe. Cette manière de faire concerne douze pièces. 

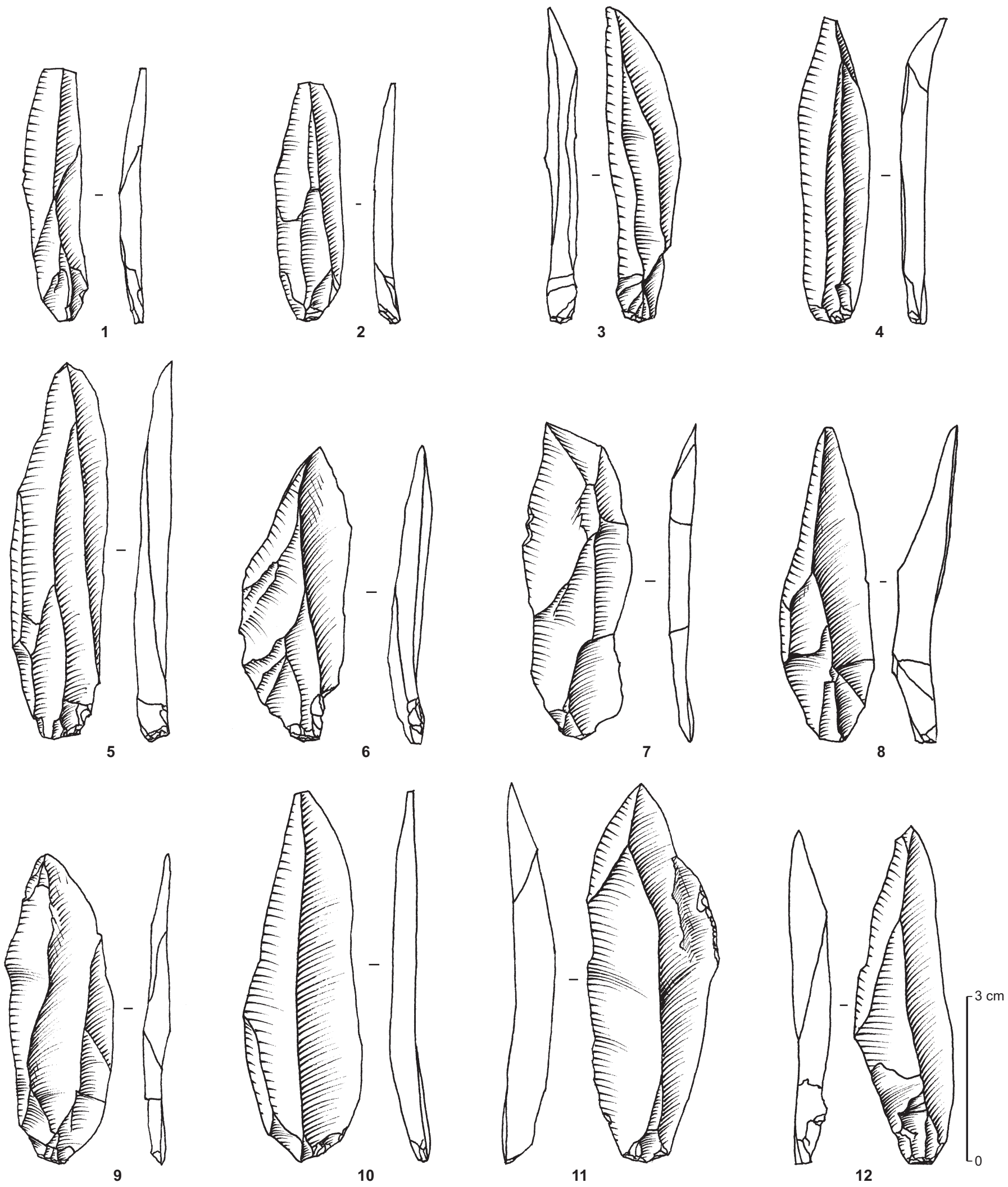

Fig. 2 - Supports bruts issus de la production de fléchettes (dessin: D. Pesesse). 

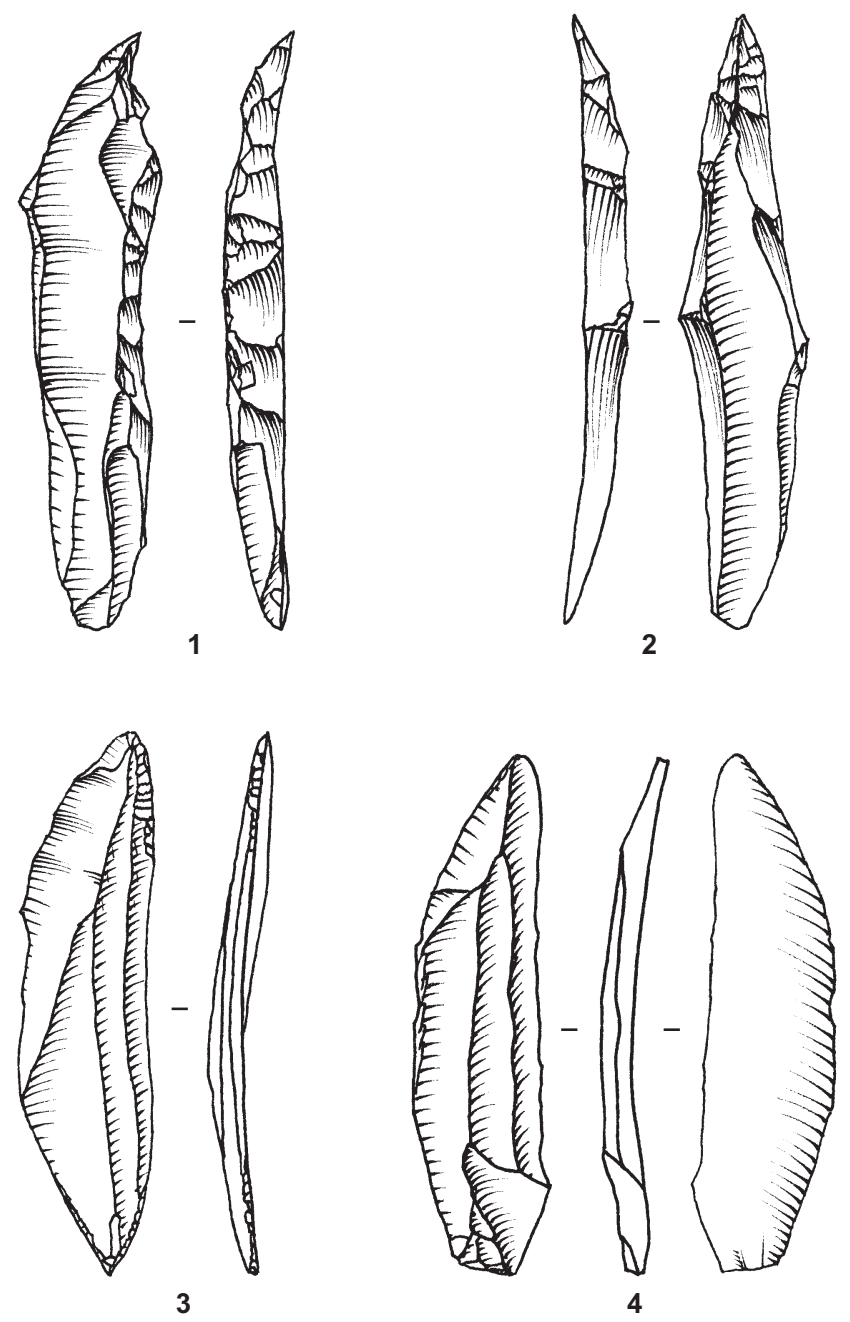

3
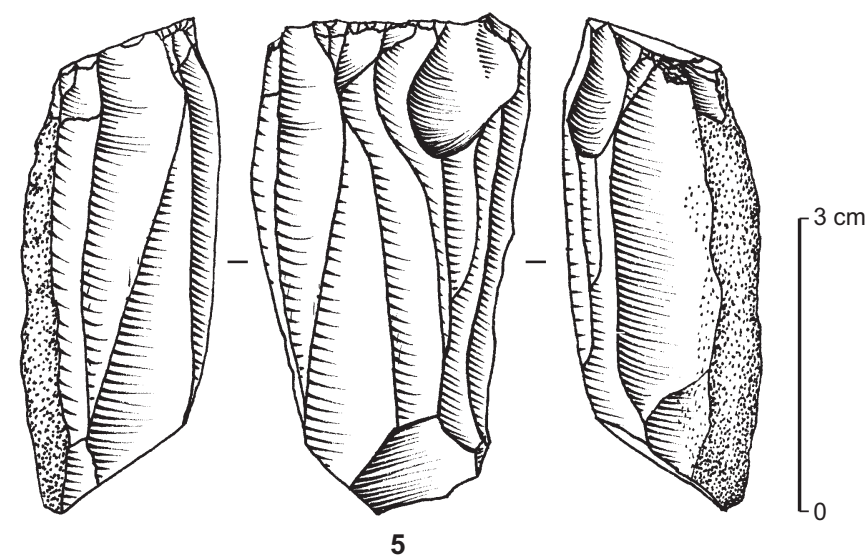

Fig. 3 - Éléments appartenant à une version simplifiée de la production de fléchettes (dessin: D. Pesesse).
En outre, vingt-huit supports sont affectés d'une retouche abrupte qui définit un dos courbe, non tranchant, sur toute la longueur, systématiquement latéralisé à droite (fig. 5).

Ces deux modes de transformation présentent des constances dans les objectifs sous-jacents. Dans chaque cas, la direction, l'intensité, la délinéation et la latéralisation de la retouche sont systématiques. Compte tenu de ces récurrences et de l'homogénéité de chaque groupe, ces variations peuvent donc être interprétées comme des normes. Sur les 179 fléchettes s'individualisent alors 12 fléchettes asymétriques ou fléchettes à dos droit et 28 fléchettes à dos courbe ${ }^{2}$. Les supports sélectionnés pour les fléchettes à dos droit sont identiques aux supports de fléchettes. En revanche, les fléchettes à dos courbe se singularisent par une certaine largeur.

F. Lacorre rassemble ces deux sous-types sous l'appellation «fléchettes à un bord abattu courbe et talon en pointe » (Lacorre, 1960). Ces spécimens correspondent en partie aux sous-types E, E' et F de H. Delporte (1972).

\section{LA FLÉCHETTE}

La discrimination de ces deux objectifs autorise donc à reconsidérer les 139 pièces répondant véritablement à la définition de la fléchette (fig. 6 et fig. 7). L'important panel d'outils entiers offre des conditions favorables à l'observation des modes d'aménagement (tabl. II).

Les 115 outils entiers ou subentiers révèlent la prépondérance de la retouche directe dans l'aménagement (tabl. III). Elle affecte $61,7 \%$ des bases et $66,1 \%$ des apex. Les variations morphologiques du support impliquent un recours ponctuel à une retouche inverse, alterne ou combinant plusieurs modes.

La morphologie des produits étant nettement favorable, l'appointement est d'extension limitée dans la majorité des cas. Pour 9 pièces seulement, la retouche reprend l'ensemble du bord pour définir la délinéation convexe recherchée (fig. 7, no 2). Majoritairement semi-abrupte, elle réduit très peu la largeur initiale. La finesse de la partie distale n'implique pas un amincissement accru par une retouche rasante. Une seule pièce a bénéficié d'une retouche apicale bifaciale particulièrement soignée (fig. $7, \mathrm{n}^{\circ} 6$ ). En tout, 24 parties

2. Pour F. Lacorre, ces pièces à dos courbe signent l'appartenance de ce phylum au Périgordien II, dans la lignée châtelperronienne. Certaines pièces de ce niveau sont d'ailleurs marquées " Périgordien II ». En effet, Lacorre n'observe aucune solution de passage du Bayacien au Gravettien, et leur parenté provient de leur origine châtelperronienne commune (Lacorre, 1960). 

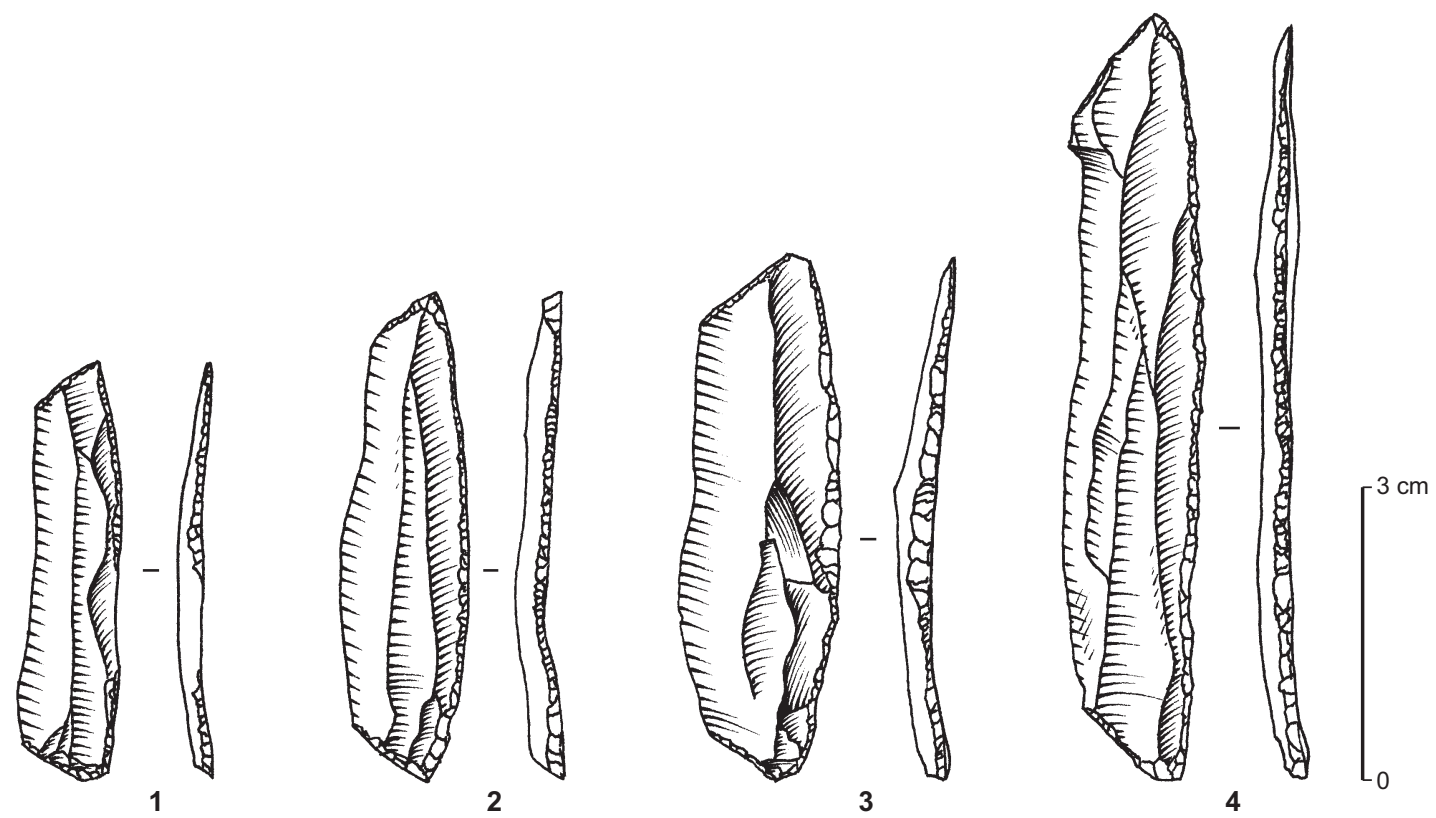

Fig. 4 - Fléchettes asymétriques (dessin : D. Pesesse).
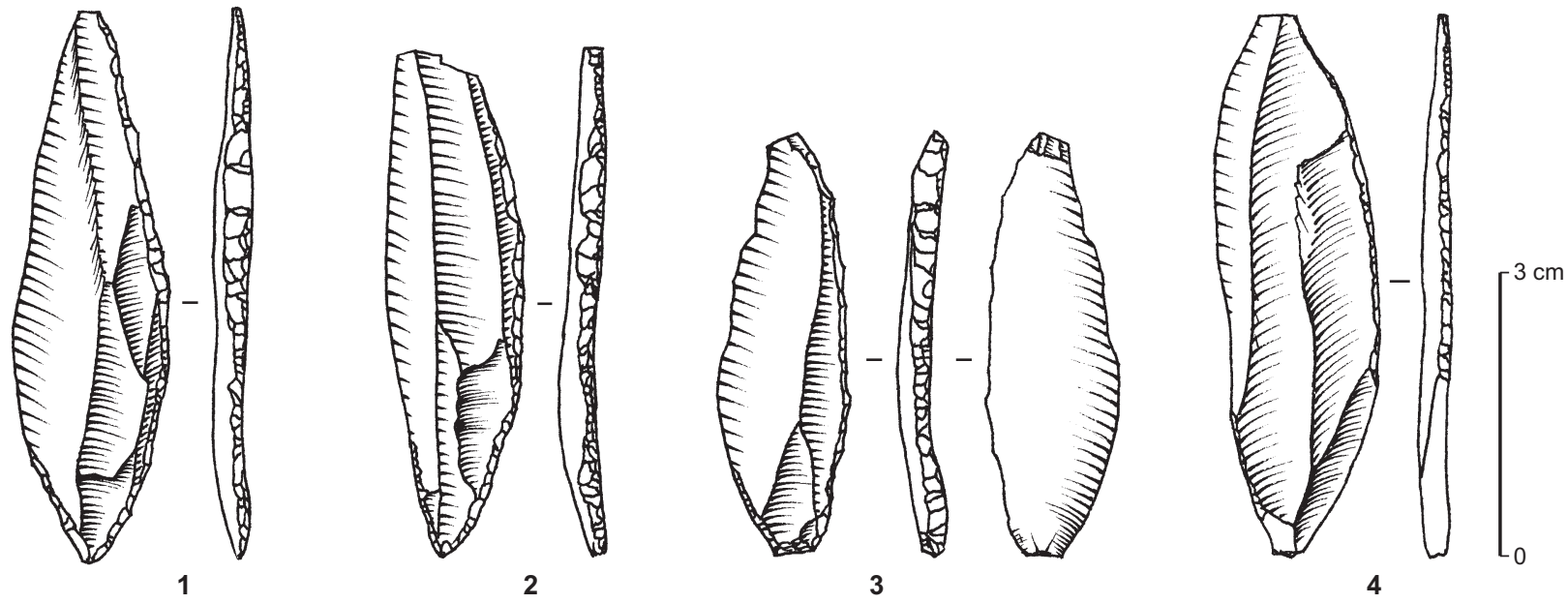

Fig. 5 - Fléchettes à dos courbe (dessin : D. Pesesse).

apicales (sur 139 outils) ont été redressées par une retouche inverse. La base est définie selon une morphologie triangulaire s'évasant progressivement.

La longueur des fléchettes s'échelonne entre $26 \mathrm{~mm}$ et $73 \mathrm{~mm}$ (sur 115 supports entiers). Les supports mesurant plus de 60-65 mm correspondent aux produits issus des premières phases du débitage, qui s'arrête aux alentours de 30-35 mm. Les produits compris entre $35 \mathrm{~mm}$ et $60 \mathrm{~mm}$ constituent le cœur de la production (tabl. IV). Leur largeur se situe entre $7 \mathrm{~mm}$ et $18 \mathrm{~mm}$ (sur 139 supports) (tabl. V), avec une moyenne de $12 \mathrm{~mm}$ (tabl. VI). 111 supports mesurent entre $2 \mathrm{~mm}$ et $3 \mathrm{~mm}$ d'épaisseur (tabl. VII). L'élancement (rapport longueur moyenne/ largeur moyenne) de l'outil diminue progressivement au cours de la réduction (tabl. VIII). Sur ces bases techniques et dimensionnelles, un unique module de fléchette peut être mis en évidence.

Contrairement à ce qui était évoqué (Delporte, 1972; Perpère, 1992), un type précis et standardisé morphologiquement se dessine ainsi. Cet outil présente toujours une 

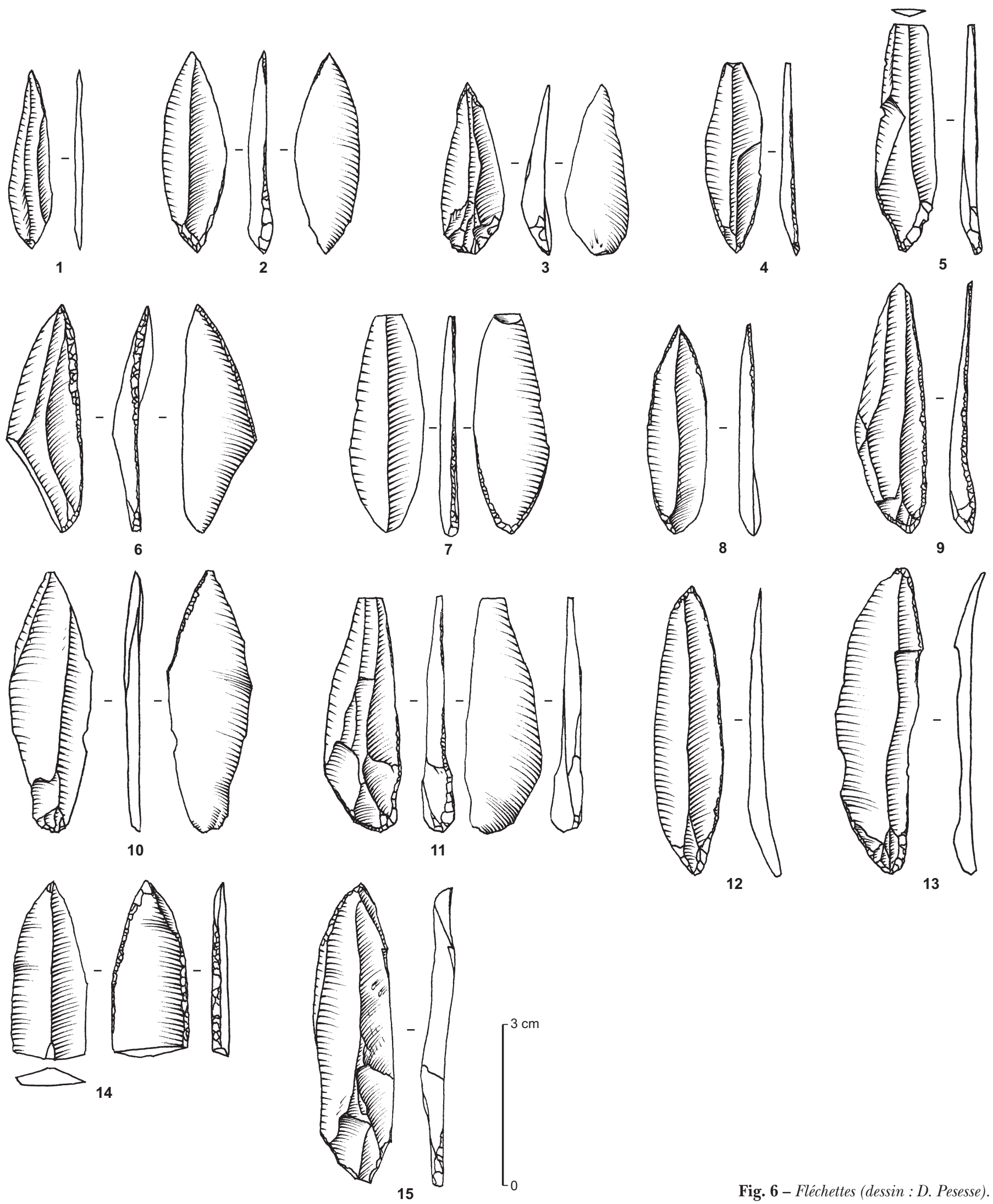

Fig. 6 - Fléchettes (dessin : D. Pesesse). 

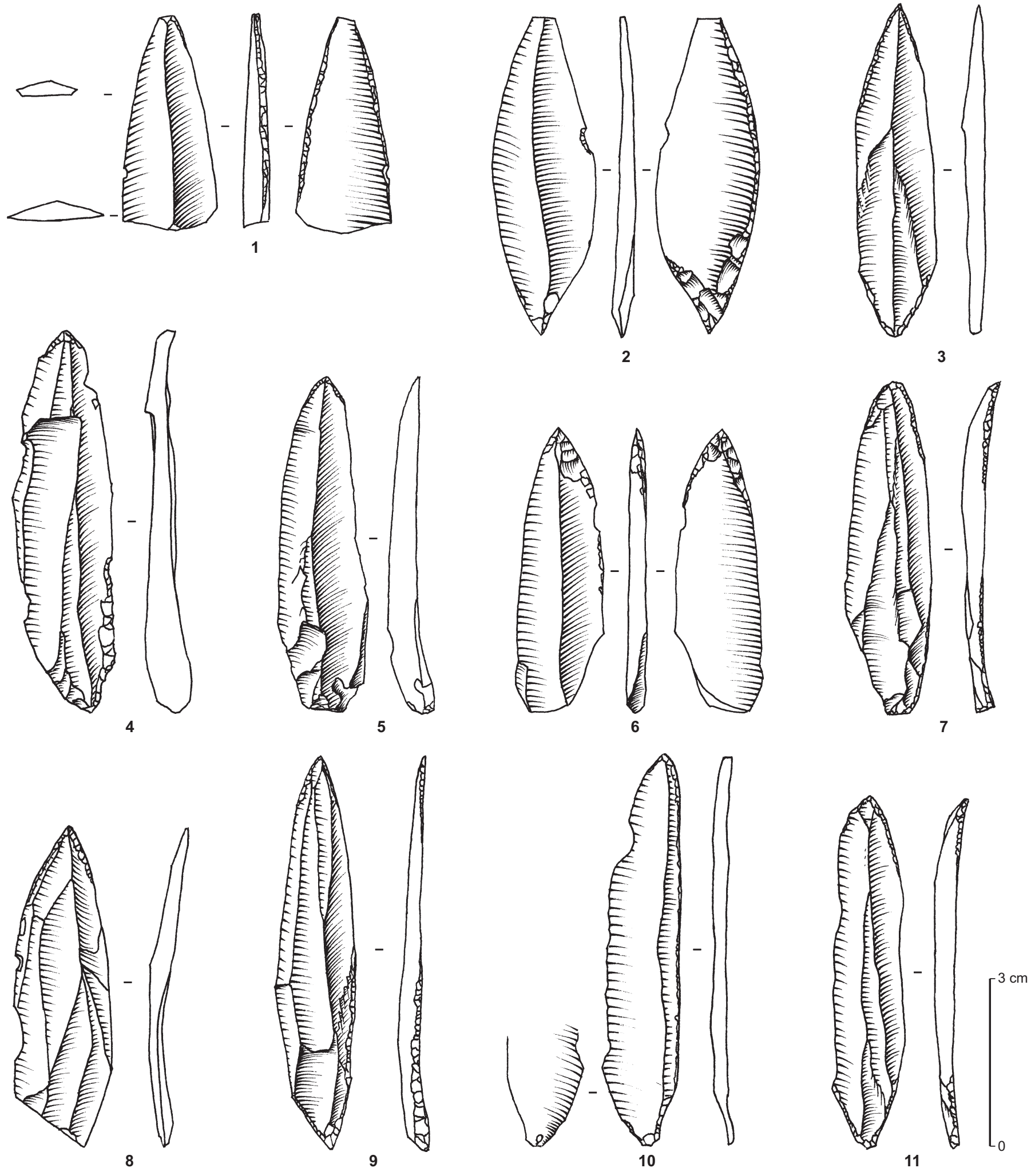

Fig. 7 - Fléchettes (dessin : D. Pesesse). 
Tabl. II - Description de la fragmentation des fléchettes.

\begin{tabular}{|l|c|c|c|}
\cline { 2 - 4 } \multicolumn{1}{c|}{} & Saint-Germain & Aurignac & Total \\
\hline Entière & 93 & 5 & $\mathbf{9 8}$ \\
\hline Subentière & 9 & 8 & $\mathbf{1 7}$ \\
\hline Fragment proximal & 1 & 2 & $\mathbf{3}$ \\
\hline Fragment mésial & 4 & 3 & $\mathbf{7}$ \\
\hline Fragment distal & 4 & 10 & $\mathbf{1 4}$ \\
\hline \multicolumn{1}{r|}{ Total } & $\mathbf{1 1 1}$ & $\mathbf{2 8}$ & $\mathbf{1 3 9}$ \\
\hline
\end{tabular}

Tabl. IV - Longueurs des fléchettes entières et subentières.

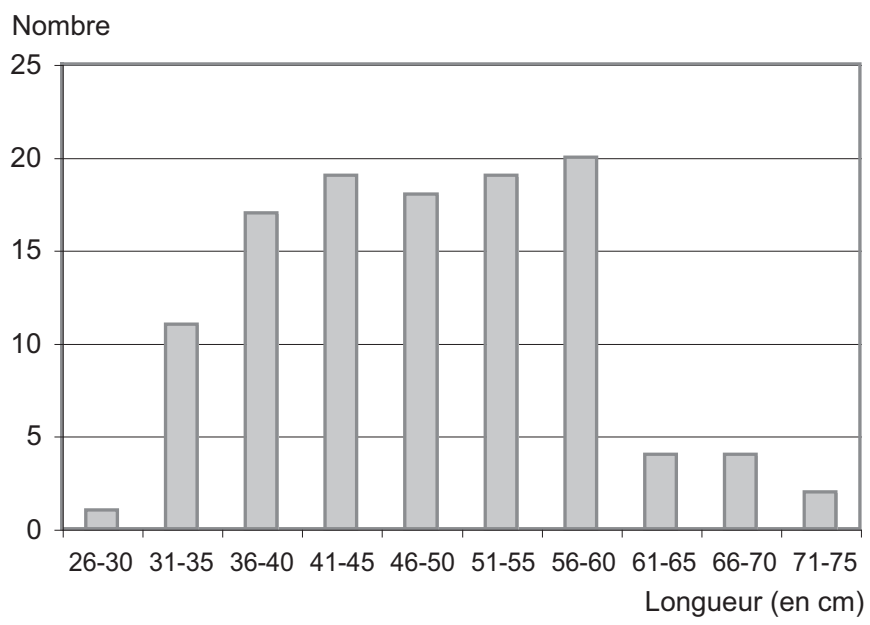

Tabl. VI - Statistiques descriptives.

\begin{tabular}{|l|c|c|c|}
\cline { 2 - 4 } \multicolumn{1}{c|}{} & $\begin{array}{c}\text { Longueur } \\
(\mathbf{e n} \mathbf{~ c m})\end{array}$ & $\begin{array}{c}\text { Largeur } \\
(\mathbf{e n} \mathbf{~ c m})\end{array}$ & $\begin{array}{c}\text { Épaisseur } \\
(\mathbf{e n} \mathbf{~ c m})\end{array}$ \\
\hline Mode & 43 & 12 & 3 \\
\hline Médiane & 48 & 12 & 3 \\
\hline Moyenne & 48,17 & 11,95 & 2,79 \\
\hline Écart type & 9,88 & 2,23 & 0,80 \\
\hline
\end{tabular}

Tabl. VIII - Variation de l'élancement des fléchettes selon la classe dimensionnelle considérée.

\begin{tabular}{|l|c|c|c|}
\hline Classes de longueurs & $30-39$ & $40-49$ & $50-59$ \\
\hline Nombre de supports & 25 & 37 & 39 \\
\hline Élancement & 3,53 & 3,89 & 4,28 \\
\hline
\end{tabular}

Tabl. III - Orientation de la retouche des fléchettes entières et subentières.

\begin{tabular}{|c|c|c|c|c|c|c|}
\cline { 3 - 7 } \multicolumn{2}{c|}{} & \multicolumn{5}{c|}{ Apex } \\
\cline { 3 - 8 } \multicolumn{2}{c|}{} & inverse & directe & alterne & brut & Total \\
\hline \multirow{4}{*}{ Base } & inverse & 15 & - & 2 & - & 17 \\
\cline { 2 - 7 } & directe & 8 & 62 & 6 & - & $\mathbf{7 6}$ \\
\cline { 2 - 7 } & alterne & - & 7 & 6 & 1 & 14 \\
\cline { 2 - 7 } & brute & 1 & 2 & 5 & - & $\mathbf{8}$ \\
\cline { 2 - 8 } & Total & $\mathbf{2 4}$ & $\mathbf{7 1}$ & $\mathbf{1 9}$ & $\mathbf{1}$ & $\mathbf{1 1 5}$ \\
\hline
\end{tabular}

Tabl. V - Largeurs des fléchettes entières et des fragments.

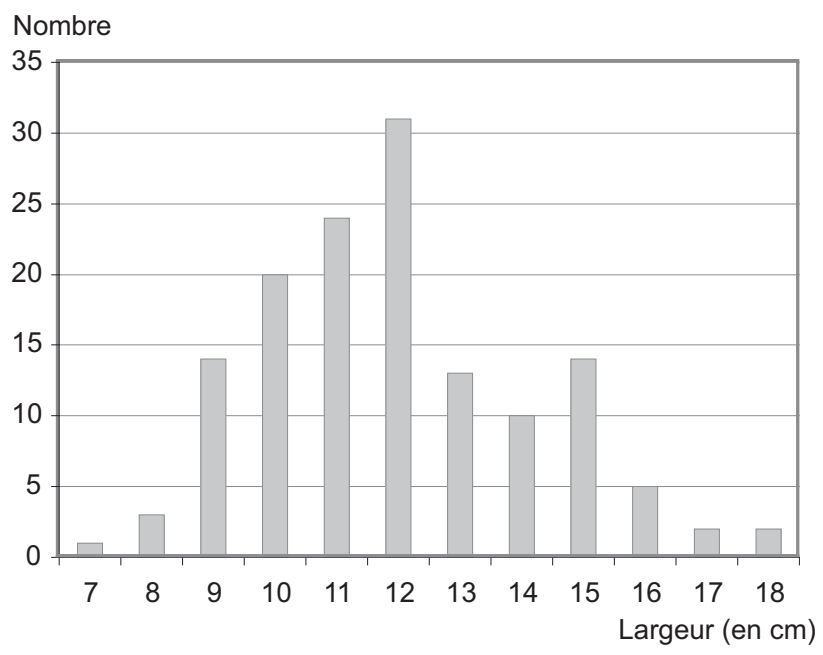

Tabl. VII - Épaisseurs des fléchettes entières et des fragments.

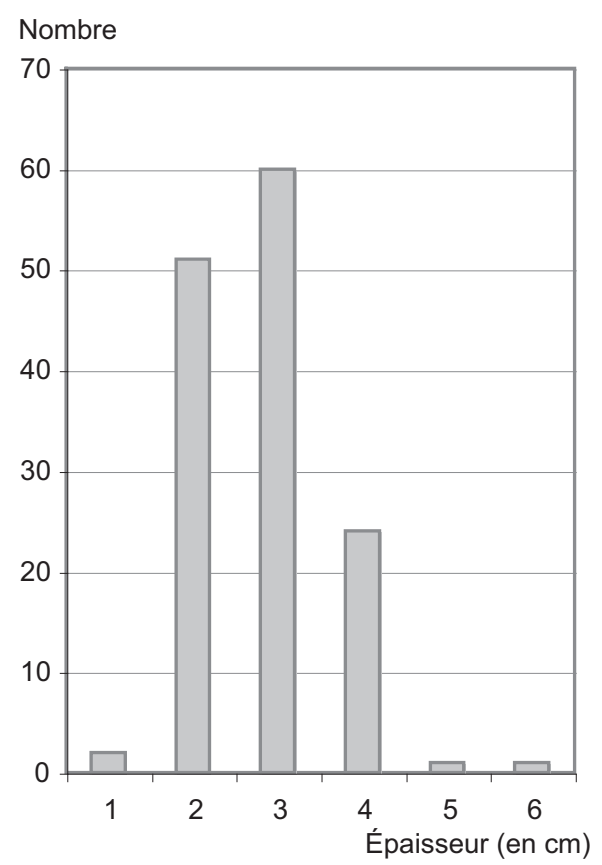



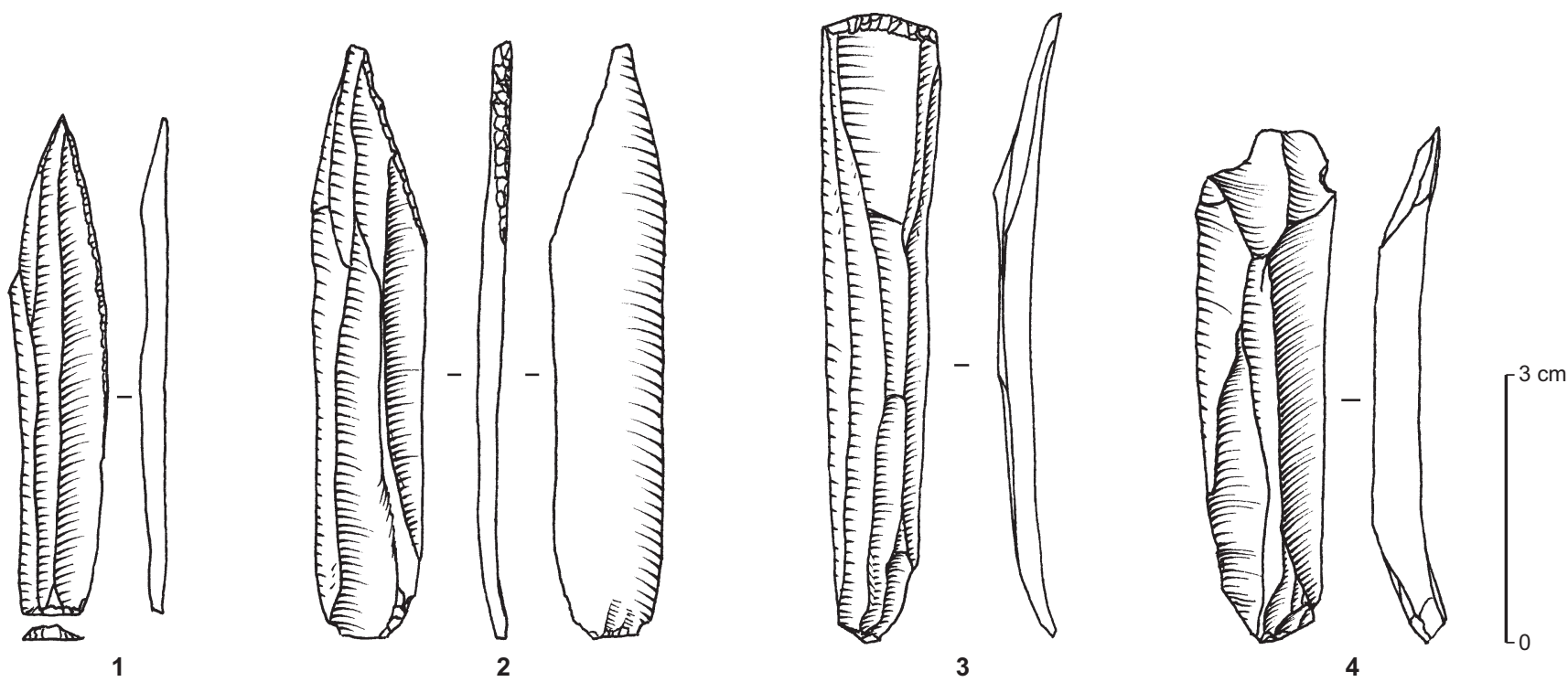

Fig. 8 - Supports vraisemblablement issus de la production bipolaire de petites lames (dessin: D. Pesesse).

grande finesse, une bonne ou très bonne régularité, un élancement important (fig. 6 et fig. 7). Sa silhouette varie d'une morphologie sensiblement laminaire (fig. $6, \mathrm{n}^{\circ} 5$ ) à des exemplaires nettement sublosangiques (fig. $6, \mathrm{n}^{\circ} 2$ ).

La fléchette peut alors être définie comme un outil conçu selon une symétrie axiale, de morphologie sublosangique, à extrémités appointées par une retouche marginale à semi-abrupte, majoritairement directe, sinon inverse ou alterne, décroissante, accentuant la convexité des bords, réalisée sur support rectiligne et polarisée.

L'existence d'une telle norme assoit la distinction des deux sous-types qui se distinguent du standard, pour l'un dès la sélection des supports, pour les deux par un mode de retouche différent, précis et constant. Ces options impliquent alors des propriétés fonctionnelles particulières. Ces sous-types illustrent la manière dont les artisans déclinent leur conception de l'outil dans sa forme principale mais également dans des variations sur le thème qui prennent très vite des caractères suffisamment marqués, distincts et récurrents pour définir des sous-types originaux. Cette manière de faire trouve un écho auprès des pointes de la Gravette du Gravettien ancien (Pesesse, 2006).

Légèreté, régularité, symétrie, bords tranchants, apex aigu, base étroite et standardisation, sont les caractéristiques configurées et accrues par l'ensemble des composantes de la production et de la transformation. Peu d'outils possèdent une adéquation aussi nette entre propriétés perforantes et tranchantes. Ces propriétés morphotechni- ques orientent l'interprétation de la fonction des fléchettes vers des armatures axiales. Compte tenu de la surreprésentation des éléments entiers, peu de stigmates permettent de confirmer cette hypothèse. Deux pièces présentent les traces d'une reprise après cassure en partie proximale et après un enlèvement burinant apical (fig. 6, no 14). En revanche, le fonctionnement des fléchettes asymétriques et à dos courbe ne peut être abordé au même degré. Ces outils révèlent la recherche d'un bord tranchant opposé à un dos fin associé à une pointe pour les premières, un tranchant convexe opposé à un dos abrupt et courbe pour les secondes.

\section{LE PETIT LAMINAIRE}

En marge de cette production principale, de rares lames se dissocient de cette norme (fig. 8). Elles correspondent à de petits supports rectilignes, sveltes, élancés, à bords parallèles. Ces lames proviennent de nucléus $(\mathrm{N}=2)$ dont les caractéristiques architecturales s'avèrent foncièrement différentes des précédents. La table de ces nucléus est longue, étroite et délimitée latéralement par des flancs abrupts, lisses et parallèles (fig. 9). Le volume est géré à partir de deux plans de frappe opposés. L'investissement et la chronologie des négatifs indiquent que ces deux plans de frappe ne partagent pas les mêmes objectifs et fonctionnent de manière non synchrone. Une alternance entre l'extraction 

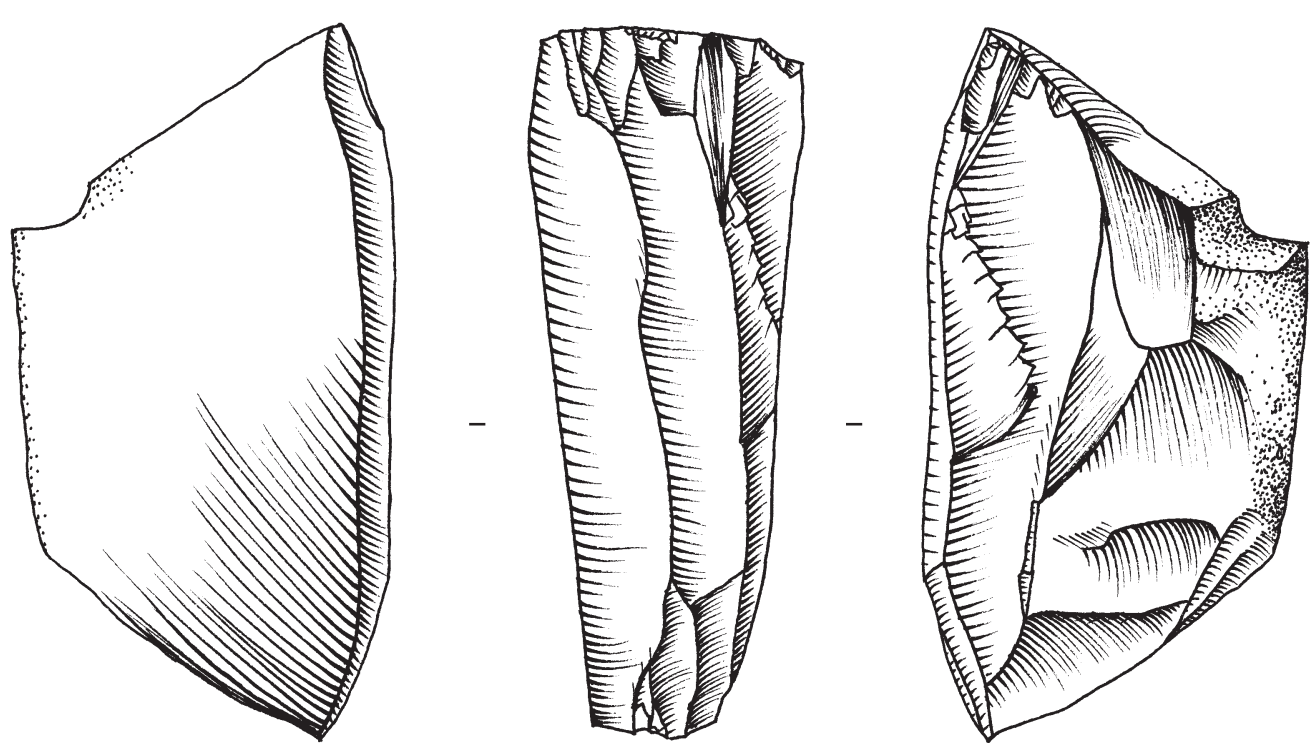

1
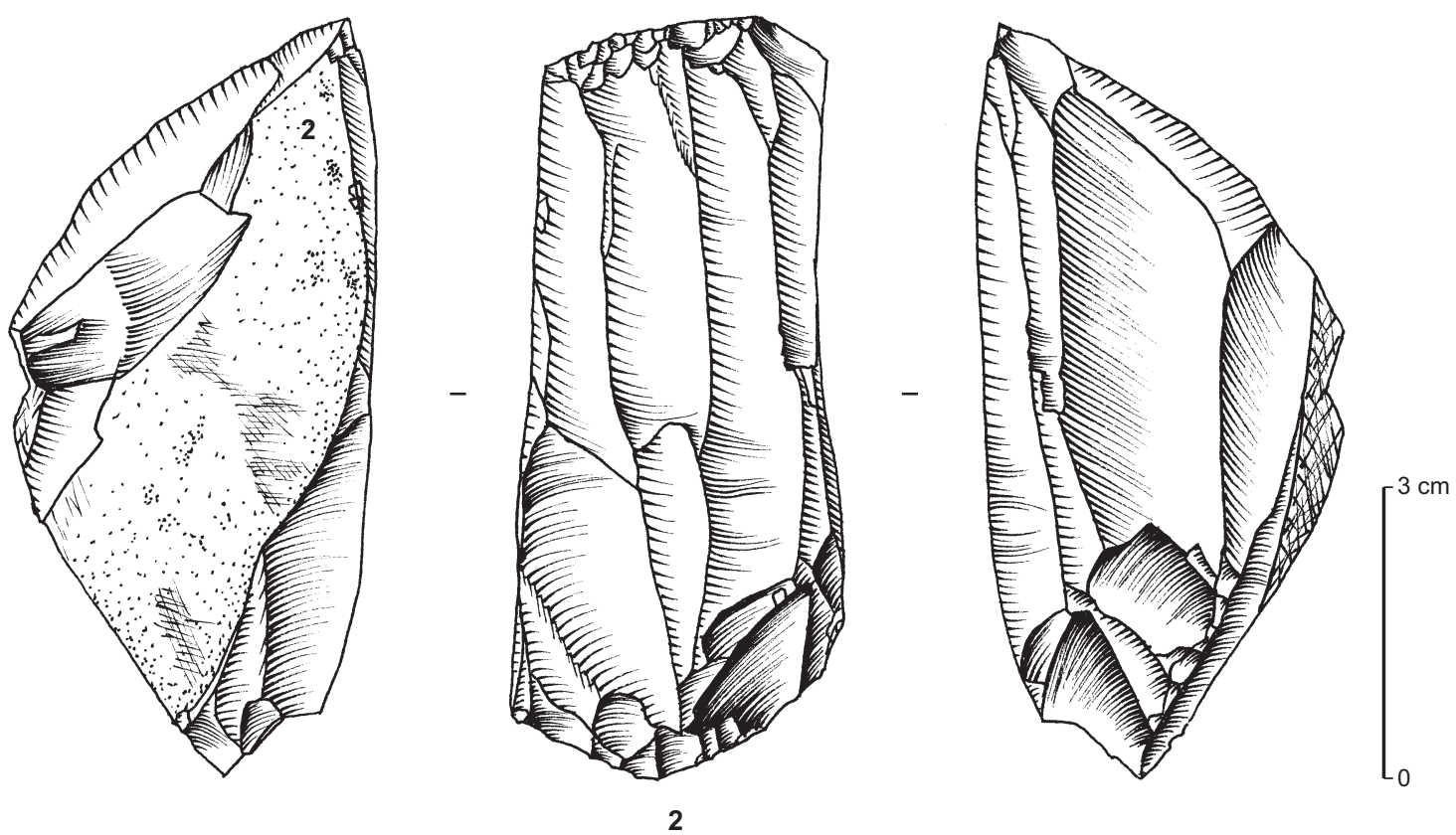

Fig. 9 - Nucléus bipolaires à petites lames (dessin : D. Pesesse).

de lames longues et rectilignes avec des supports plus courts et moins réguliers est identifiable. Ces plans de frappe sont donc hiérarchisés, l'un servant à la production stricto sensu, l'autre à l'entretien du volume.

Les produits obtenus dans ce cas sont des lames légères à bords parallèles, transformées en grattoir, et en lames appointées. Leur proportion est minoritaire - ou sousreprésentée - dans l'assemblage.

Ce mode opératoire s'écarte donc du schéma précédent, car les produits présentent des caractéristiques morphologiques distinctes par leur élongation, leur délinéation et vraisemblablement leur transformation. La sélection de volumes 
étroits (plaquette et éclat) écarte la possibilité d'un continuum avec le débitage de fléchettes, dont le galbe du volume est une condition sine qua non. En revanche, ils partagent la même distinction fonctionnelle des plans de frappe.

\section{LE GRAND LAMINAIRE}

Représentée par 62 éléments, une fraction laminaire se démarque fortement des ensembles précédents (fig. 10). Le premier élément fédérant est le caractère majoritairement unipolaire du débitage. Seules deux lames portent les négatifs d'enlèvements opposés. L'exploitation s'amorce par une crête antérieure à un versant dans deux cas, à deux versants successifs dans un cas. Peu d'éléments latéraux trahissent les vestiges d'une mise en forme poussée (tabl. IX). $\mathrm{Au}$ contraire, la présence de lames corticales plaide en faveur d'un faible aménagement de la structure initiale. Le volume est entretenu par le débordement progressif des produits. Les lames latérales présentent une certaine diversité d'inclinaison vers les flancs. Ces lames sont extraites dans l'axe de la table ou de manière désaxée, vers la table. L'existence d'une crête postérieure est déduite de la présence d'une sous-crête et d'une sous-néocrête postérieures. Cette crête intervient donc durant le débitage. La transformation souvent distale des lames occulte partiellement la lecture de la morphologie basale des nucléus. Certaines présentent une rupture de profil en partie distale qui peut être mise en relation avec l'entretien de la carène. La réalisation d'une néocrête en partie distale de lame peut relever de la même optique.

Les lames obtenues sont relativement massives, parfois de belle facture; celles extraites au centre de la table présentent une certaine régularité par le parallélisme des bords et des nervures, la symétrie des sections et donc la délinéation régulière des tranchants. Toutefois, peu de constances opératoires sont observables, ce dont témoigne la relative diversité morphologique des produits obtenus. Sur les huit parties proximales observables, les quatre talons diagnostiques se rattachent à l'utilisation de la percussion organique.

La distinction produit/sous-produit paraît peu stricte, bien que la majorité des outils soit réalisée sur des lames extraites au centre d'une table régulière. Ces lames sont transformées essentiellement en burins (26), puis en grattoirs (14), en outils mixtes (4), enfin en divers outils, dont perçoirs (2), lames tronquées ou simplement retouchées (11).
Si deux nucléus présentent des caractéristiques laminaires, un seul semble correspondre aux objectifs de la production. Ce nucléus, de section quadrangulaire et exploité de manière unipolaire, a subi plusieurs réorientations de tables juxtaposées. Le second nucléus, ainsi qu'une lame latérale, traduisent l'exploitation d'une surface très plate, délimitée par une corniche périphérique. Cette structuration volumétrique très particulière isole fortement ce nucléus et cette lame latérale au sein de cet assemblage.

\section{UN ASSEMBLAGE HOMOGÈNE?}

La question de l'intégrité de ce niveau tient une place prépondérante dans cette problématique, car vérifier l'originalité de cet assemblage conduit à tester le sens imparti au «Bayacien ».

Durant la fouille, F. Lacorre a vraisemblablement opéré une sélection des artefacts. Ce tri affecte plusieurs catégories d'objets : les éléments bruts, les déchets, la fraction fine et les fragments d'outils. Ces biais perturbent à différents degrés la compréhension de cet ensemble.

Les données économiques, concernant la place de chaque matériau ou le mode d'introduction des nucléus, sont largement tronquées. De même, la sous-représentation des fragments de fléchettes ne permet pas d'appréhender précisément le mode de fonctionnement de cet outil, bien que ses paramètres morphofonctionnels traduisent vraisemblablement une fonction d'armature. La qualité de la lecture technologique en souffre également, en particulier sur la sélection des supports, les modes d'initialisation, le rythme de l'alternance des plans de frappe opposés et les modalités de percussion. La précision dans la caractérisation de chaque schéma dépend alors de sa représentation quantitative au sein de la collection. En dépit de ces lacunes, la résolution atteinte dans la caractérisation de ces productions révèle la complexité des processus employés, tout particulièrement pour les fléchettes, et accrédite la cohérence des procédés mis en évidence. Les imprécisions concernent donc principalement le rôle du schéma bipolaire et les modalités de la production laminaire de grand module.

Les biais liés à l'ancienneté de la fouille mis en évidence compromettent donc principalement la compréhension de la fonction de l'occupation et certains points précis de l'analyse technologique. L'originalité de cette industrie transparaît en dépit de ces biais. Il convient à présent d'évaluer sa spécificité en la comparant avec les systèmes techniques documentés dans les autres niveaux du gisement. 

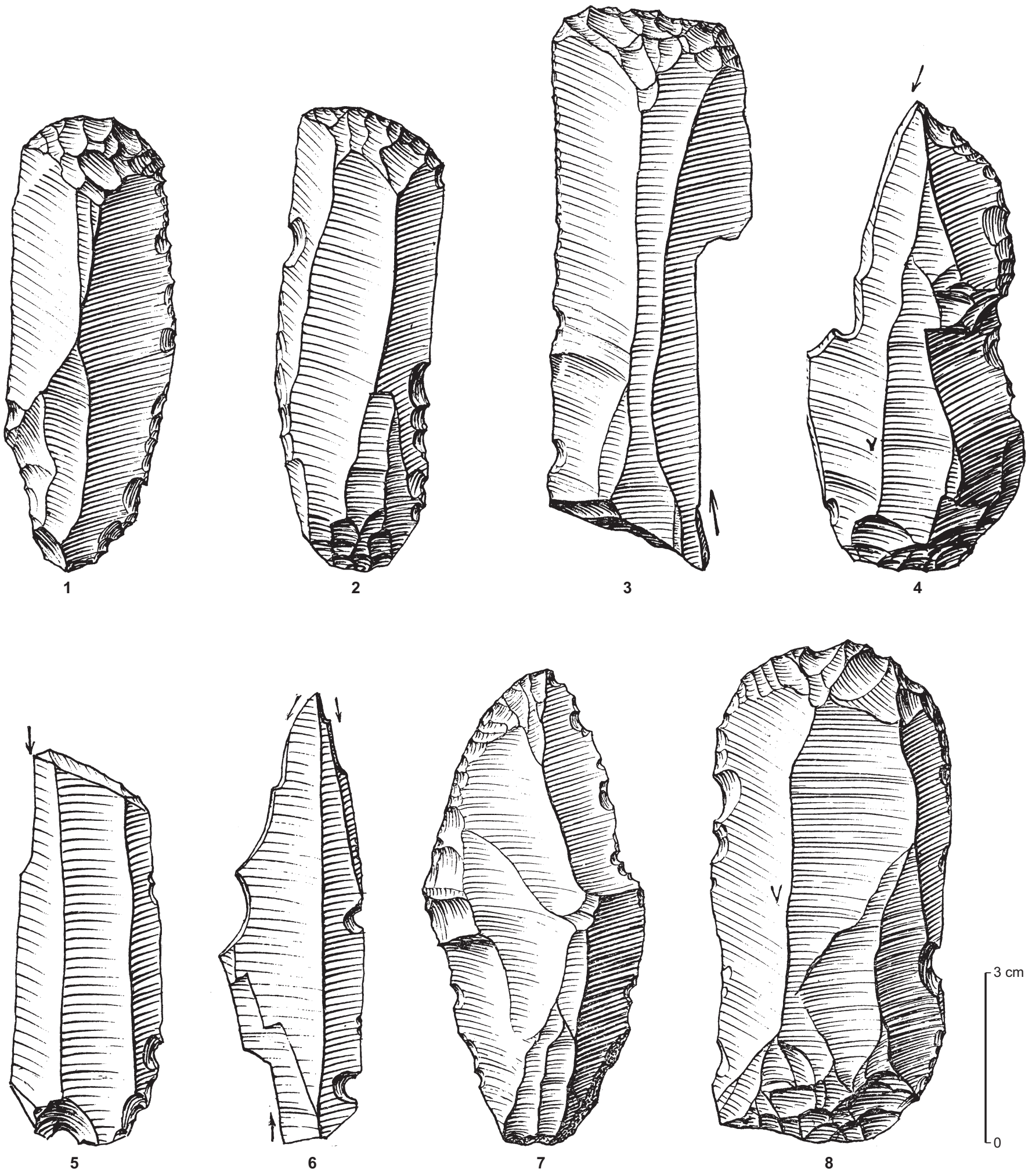

Fig. 10 - Outils sur lames (dessins J. Bouyssonie, in Lacorre, 1960). 
Tabl. IX - Description technologique de la série laminaire de grand module.

\begin{tabular}{|c|c|c|c|c|c|}
\hline & \multicolumn{2}{|c|}{ Nature } & \multirow{2}{*}{$\begin{array}{c}\text { Brut } \\
-\end{array}$} & \multirow{2}{*}{$\begin{array}{c}\text { Retouché } \\
3\end{array}$} & \multirow{2}{*}{$\begin{array}{c}\text { Total } \\
\mathbf{3} \\
\end{array}$} \\
\hline \multirow{9}{*}{ LAME } & \multirow{2}{*}{ à crête } & à un versant & & & \\
\hline & & à deux versants & 1 & 1 & 2 \\
\hline & \multirow{2}{*}{ sous-crête } & antérieure & - & 4 & 4 \\
\hline & & postérieure & 1 & - & 1 \\
\hline & \multirow{2}{*}{ d'entretien } & des flancs & 3 & 2 & 5 \\
\hline & & de la carène & 1 & 3 & 4 \\
\hline & \multicolumn{2}{|l|}{ néocrête } & 1 & 1 & 2 \\
\hline & \multicolumn{2}{|c|}{ sous-néocrête } & 1 & - & 1 \\
\hline & \multicolumn{2}{|c|}{ de plein débitage } & 1 & 26 & 27 \\
\hline \multirow{5}{*}{ ÉCLAT } & \multicolumn{2}{|l|}{ laminaire } & - & 10 & 10 \\
\hline & \multicolumn{2}{|l|}{ cortical } & - & 5 & 5 \\
\hline & \multicolumn{2}{|l|}{ indéterminé } & 2 & 3 & 5 \\
\hline & \multicolumn{2}{|l|}{ tablette } & 1 & 1 & 2 \\
\hline & & Total & 12 & 59 & 71 \\
\hline
\end{tabular}

Les productions aurignaciennes, réalisées en grande majorité en silex sénonien, correspondent à des débitages lamellaires sur grattoirs à museaux et burins busqués, ce qui réduit considérablement les possibilités de contamination. Aucune intention lamellaire n'est d'ailleurs attestée avec certitude pour le Bayacien. La production laminaire aurignacienne, hormis les pièces introduites en Bergeracois, se distingue également par sa faible régularité et l'utilisation ponctuelle de la percussion dure pour détacher des lames de flancs.

Les productions gravettiennes, très homogènes entre les différentes couches, affirment une forte originalité liée à la mobilisation de savoir-faire complexes destinés à produire des supports de gravettes de différents modules. À côté des pointes de la Gravette coexistent de nombreux types d'armatures, pointes de Tursac, pointes à crans... Les différences concernent donc à la fois la structuration de l'industrie, les normes techniques, les objectifs fonctionnels et le module des supports obtenus, dans un continuum dimensionnel de $200 \mathrm{~mm}$ à $50 \mathrm{~mm}$. Une production de lamelles sur tranche d'éclat est également attestée.

Dans leurs caractéristiques fondamentales, les productions aurignaciennes, bayaciennes et gravettiennes relèvent de normes suffisamment distinctes pour entériner une discrimination d'ordre technologique.

Sur ces bases, certaines pièces de ce niveau se démarquent nettement des normes bayaciennes: un burin busqué sur lame, un éclat de ravivage latéral de pièce carénée (Lacorre, 1960, pl. XVI, no 158), quelques lames larges et rectilignes $(\mathrm{N}=6)$, le nucléus à corniche périphérique et le support débordant mentionnés supra (Lacorre, 1960, pl. XX, no 197 et pl. XXI, no 207). Parmi ces éléments, les deux premiers peuvent donc provenir du niveau aurignacien, les quelques lames du Gravettien sus-jacent et les deux derniers sont indéterminés. Les modes opératoires bayaciens sont donc suffisamment élaborés pour exclure les éléments isolés techniquement dans ce niveau, mais répondant à des normes identifiées dans les niveaux souset sus-jacents, et donc vraisemblablement intrusifs. Pour un gisement fouillé à partir des années 1930, les contaminations sont donc minimes totalisant dix pièces, soit $3 \%$ de la série. H. Delporte constatait déjà « l'absence à peu près totale de formes typiques du Gravettien »(Delporte, 1972, p. 344).

La cohérence globale de l'assemblage peut donc être envisagée. L'ensemble fonctionne comme un système au sein duquel les objectifs sont complémentaires. La fraction laminaire s'intègre de manière fonctionnelle dans une bipartition «domestique/cynégétique » avec les fléchettes, si l'on accepte cette interprétation fonctionnelle, suggérée également à partir des pièces de l'abri Pataud (Perpère, 1992). Cette industrie originale est structurée autour de l'idée d'une armature axiale sublosangique. Elle est menée à bien par un schéma opératoire spécialisé, très investi techniquement. Cet investissement apparaît au travers du degré d'adéquation support/outil. Ce schéma opératoire ne peut se résumer à un débitage unipolaire entretenu par un plan de frappe opposé décalé. L'agencement de produits sur la table, la gestion de contraintes volumétriques exacerbées, la différenciation des plans de frappe sont le fruit de logiques opératoires complexes. Cette complexité constitue un des garants de l'originalité de cette production. Elle répond à un degré de fait la distinguant des procédés unipolaires, ubiquistes durant le Paléolithique supérieur.

\section{LE BAYACIEN D'UN POINT DE VUE HISTORIQUE}

L'ensemble des éléments contribue donc à reconnaître la spécificité du Bayacien. Par son originalité, l'industrie reconnue à la Gravette par F. Lacorre en 1930 mérite donc d'être distinguée des ensembles postérieurs. Maintenir le terme de Bayacien cristallise cette seule particularité.

À partir de cette nouvelle définition, des comparaisons peuvent être testées avec le Bayacien. L'abri Vignaud 

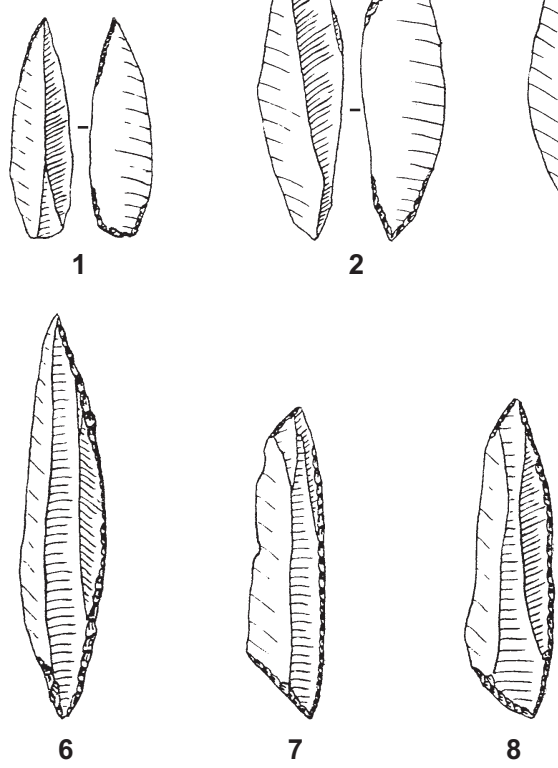

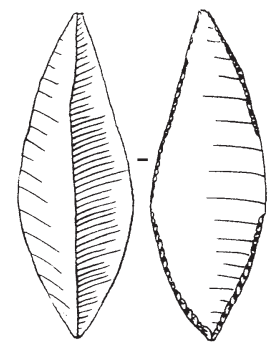

3

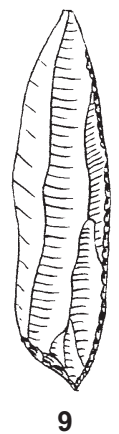

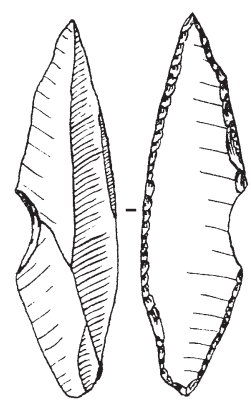

4
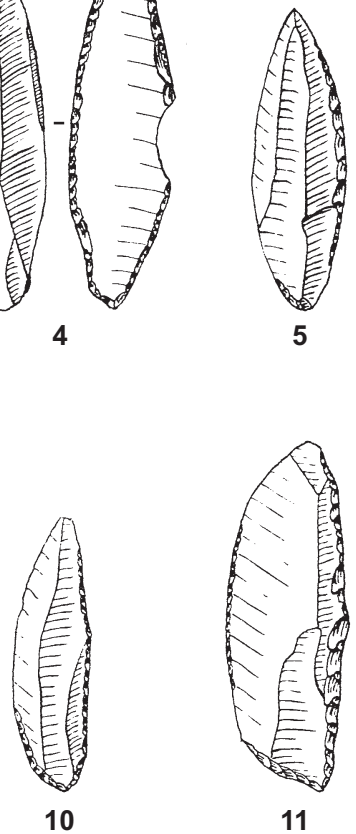

Fig. 11 - Fléchettes et fléchettes asymétriques de l’abri Vignaud aux Eyzies-de-Tayac (Dordogne). Échelle absente (d'après Sonneville-Bordes, 1960, modifié).

aux Eyzies-de-Tayac (Dordogne) est rapproché de cet horizon sur des bases typologiques (fig. 11) (SonnevilleBordes, 1960). La fléchette tient apparemment une place importante dans cette industrie. D. de Sonneville-Bordes distingue également des pièces asymétriques des fléchettes classiques (fig. 11, $\mathrm{n}^{\text {os }} 6$ à 11) (Sonneville-Bordes, 1960). Malgré la cohérence apparente de l'assemblage tel que figuré, cette série peut difficilement être prise en compte, faute de données stratigraphiques. En effet, D. Peyrony explora ce gisement, mais ne trouva que les déblais des fouilles anciennes ou des niveaux remaniés (Peyrony, 1947). La position chronostratigraphique de cet horizon demande également à être précisée ; ce niveau a d'ailleurs été retrouvé lors d'un sondage réalisé en 1981 (Geneste, 1982).

Le gisement de Pair-non-Pair à Marcamps en Gironde révèle également certaines affinités (Cheynier, Daleau, 1963). En effet, un nucléus reproduit l'ensemble des caractéristiques décrites précédemment (fig. 12, no 1). Des fléchettes particulièrement soignées sont présentes, ainsi que des fléchettes asymétriques (fig. $12, n^{\text {os }} 3$ et 8 ). Les supports utilisés témoignent toutefois d'un élancement important et le parallélisme de leurs bords les distingue quelque peu des pièces éponymes (fig. 12, nos 2 à 4). La fouille de Pair-non-
Pair s'étant déroulée à la fin du XIX ${ }^{\mathrm{e}}$ siècle, ces pièces proviennent de plusieurs horizons (niveaux 3, 4, CD, D et K).

En l'état actuel des connaissances, ces deux industries ne peuvent donc être attribuées avec certitude au Bayacien. Le niveau de la Gravette demeure original. De nombreux gisements, essentiellement nord-aquitains, ont pourtant livré des fléchettes. Mais dans ces assemblages, cet outil est toujours associé aux pointes de la Gravette dans les sites fouillés dans de bonnes conditions: couche 5 de l'abri Pataud aux Eyzies-de-Tayac en Dordogne (Bricker, 1977; Bricker dir., 1995; Leoz, 2001), Puy Jarrige à Brive-laGaillarde en Corrèze (Mazière et al., 1984), la Vigne Brun à Villerest dans la Loire (Pesesse, 2003) (fig. 13). La présence de fléchettes ne peut alors être considérée comme diagnostique du Bayacien. Le Bayacien correspond en effet à un système technique et non à un outil.

À l'heure actuelle, la présence de fléchettes, et notamment de fléchettes asymétriques (abri Vignaud et Puy Jarrige), dans l'ensemble des gisements du Gravettien ancien aquitain traduit la perduration de traditions, dont l'articulation avec le Bayacien demande encore à être définie. Les gradients de cette évolution seraient à chercher dans d'autres gisements, en particulier la couche 5 de 

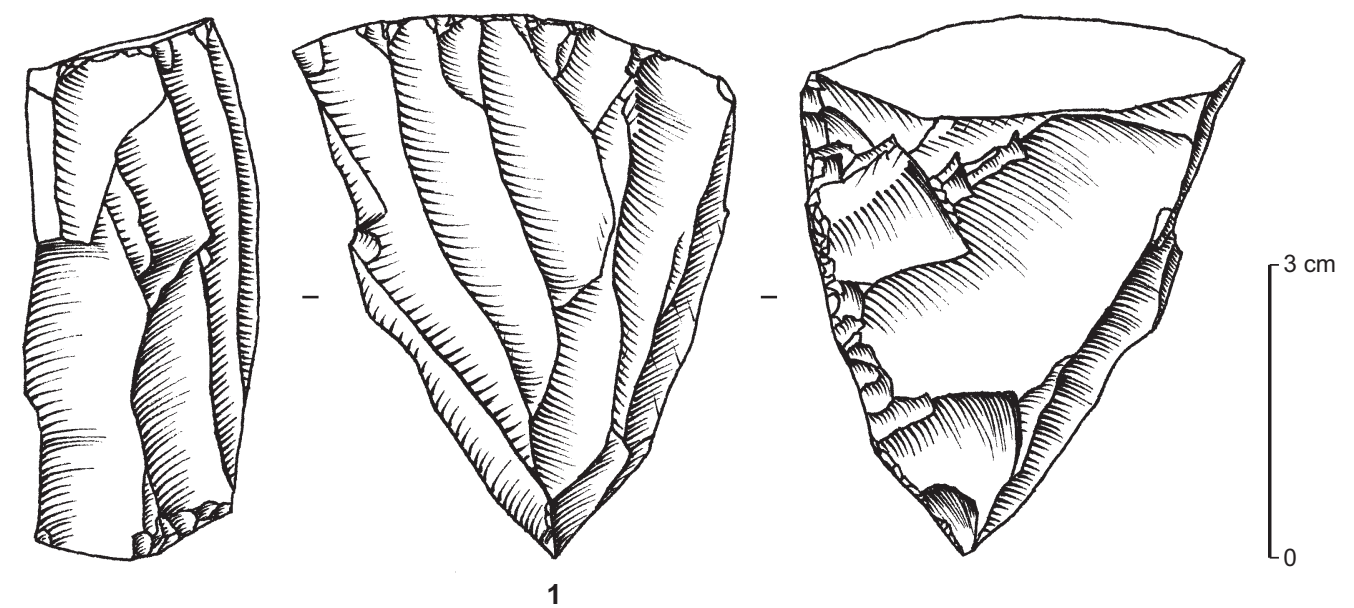

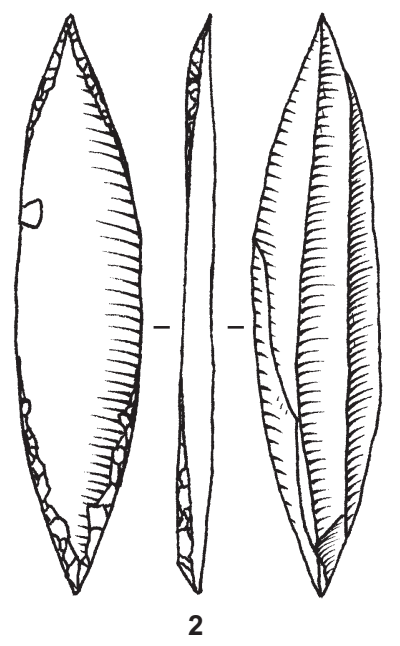

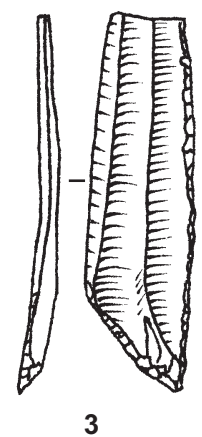

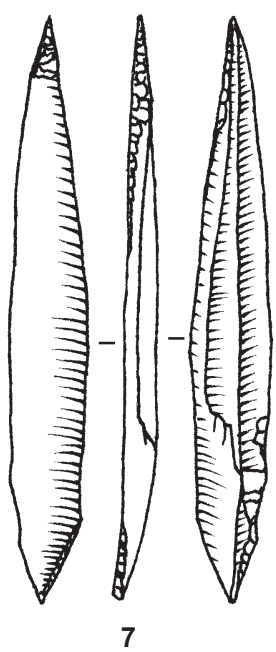

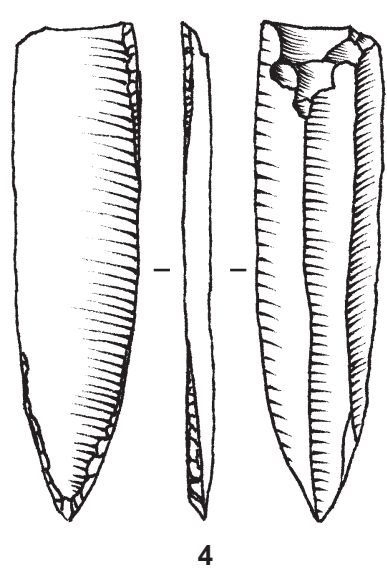
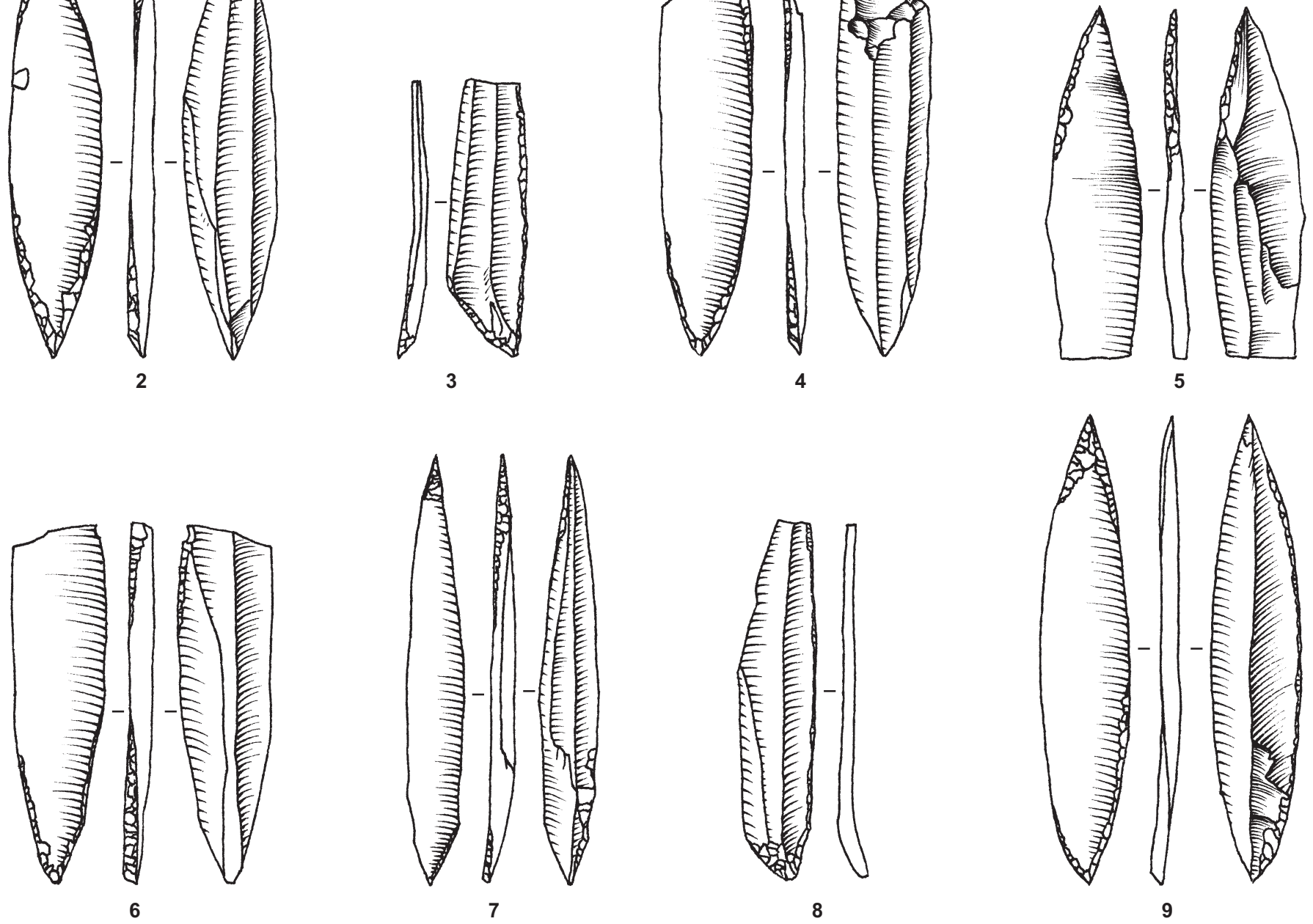

Fig. 12 - Nucléus, fléchettes et fléchettes asymétriques de la grotte de Pair-non-Pair à Marcamps (Gironde) : $1,4,7$, niveau $4 ; 2$, niveau $D ; 3,5,6$, niveau 3;8, niveau $K ; 9$, niveau CD (dessin : D. Pesesse). 


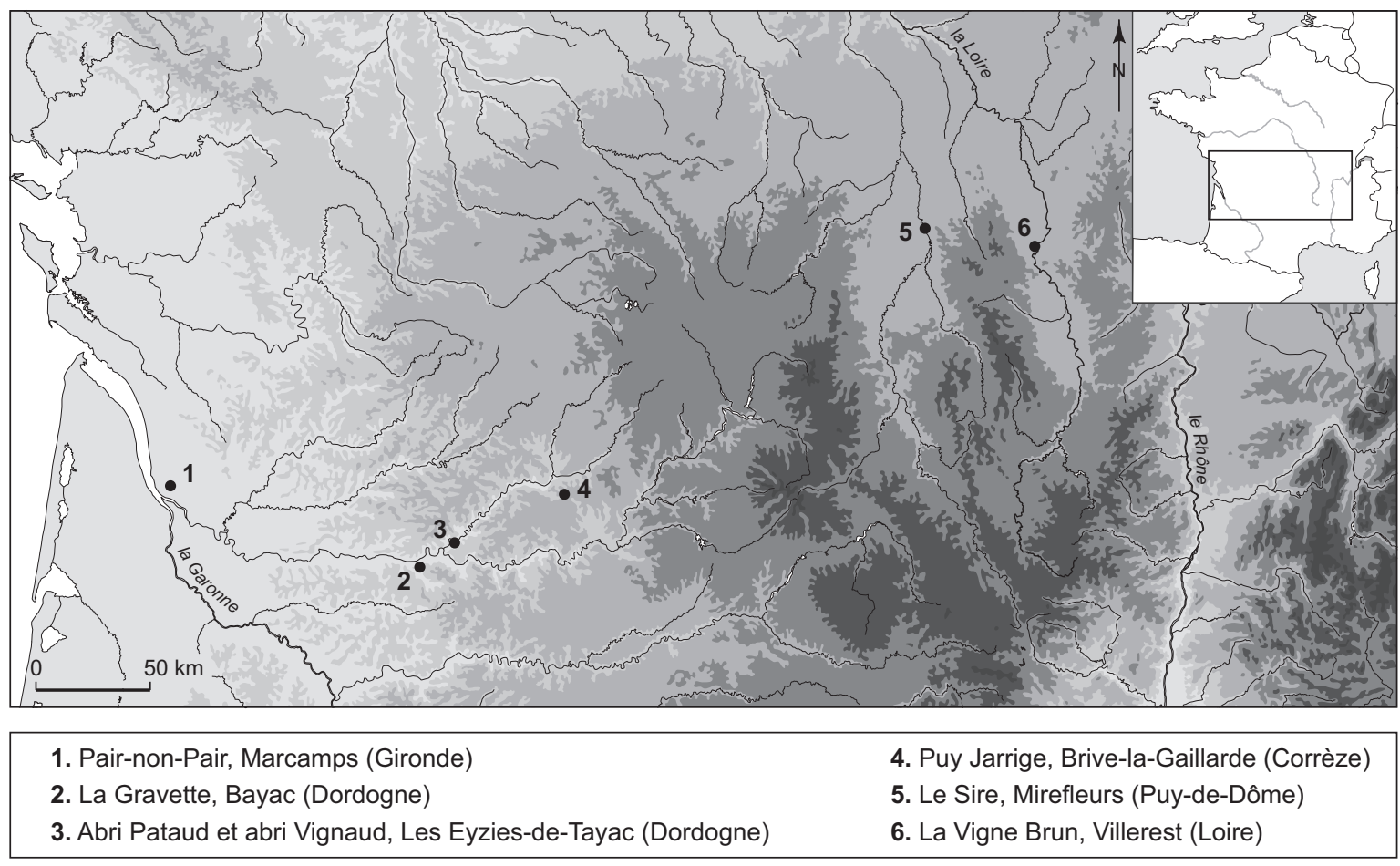

Fig. 13 - Localisation des gisements mentionnés (DAO : D. Pesesse).

l'abri Pataud (Delporte, 1972; Bricker, 1977; Perpère, 1992; Leoz, 2001). L'horizon inférieur de cette couche, Font: Lower 2, est en effet rapproché du Bayacien (Bricker, 1977), bien que les fléchettes soient associées à de nombreuses pointes de la Gravette. La reconnaissance dans ce niveau de l'association ou de l'exclusion des chaînes opératoires de production des pointes de la Gravette et de fléchettes - et leurs évolutions dans la séquence - pourraient éventuellement documenter les jalons du passage du Bayacien au Gravettien ancien. Contrairement à l'opinion de H. M. Bricker (1977), la mise en évidence de cette évolution ne doit pas masquer l'originalité de ce système technique à un moment donné.

Le caractère " gravettien » du Bayacien mérite d'être évalué au regard des horizons sus-jacents. Les procédés gravettiens s'écartent des méthodes antérieures sur la structuration globale de l'industrie, intégrant domestique et cynégétique, ainsi que sur la nature des modes opératoires mobilisés à ces fins. La prépondérance de la fléchette coïncide peu avec la diversité typologique gravettienne. Tant la structure, les normes que les objectifs différencient les industries bayaciennes et gravettiennes. Cependant, la refonte constante des systèmes de production constitue l'une des caractéristiques du Gravettien (Nespoulet, 1996; Le Mené, 1999; Digan, 2001; Klaric, 2003 et 2007; Pesesse,
2003; Goutas, 2004; Guillermin, 2004; Simonet, 2004 et 2005; Pottier, 2005).

Derrière ces différences formelles, d'autres critères sont donc à rechercher. L'apparition de la fléchette signe probablement, au-delà des modifications nécessaires du système de fonctionnement, la valorisation de l'armature axiale en silex. Dans ce processus, la pointe de la Gravette constitue le pendant conceptuel de la fléchette. L'apparition d'une tendance à la latéralisation sur un sous-type de fléchette par la réalisation de dos fins, dextres comme les pointes de la Gravette, pourrait être considérée comme les prémices à l'armature éponyme (pour cette idée, voir Demars, Laurent, 1989, p. 120). Ces éléments tissent des liens discrets entre le Bayacien et le Gravettien ancien; mais l'absence de pointes de la Gravette dans le Bayacien constitue un élément important car, dans cette optique, ce premier Gravettien n'est pas un Gravettien stricto sensu.

Cette industrie particulière nous renseigne sur ce que l'on peut considérer régionalement comme la plus ancienne manifestation du Gravettien. Cette assertion se fonde principalement sur des comparaisons stratigraphiques, car les datations des gisements rapportés au Gravettien ancien en France révèlent d'importants problèmes de résolution. En effet, ni la couche 5 de l'abri Pataud (Bricker dir., 1995), ni 
le Sire (Surmely et al., 2003), ni la Vigne Brun (Combier, 1980 et 1989; Évin, 1982), gisements se rapportant vraisemblablement à une même entité du Gravettien ancien, n'ont été datés dans une fourchette chronologique inférieure à trois millénaires chacun. L'approche par comparaisons stratigraphiques demeure alors un moyen d'accès privilégié à la sériation des premières phases du Gravettien.

Les datations des gisements «contemporains »des régions limitrophes (Jura souabe: Conard, Moreau, 2004 et Basse-Autriche), révèlent des écarts équivalents, lorsque leur calibration n'est pas pondérée par une approche chronostratigraphique élargie (Haesaerts et al., 1996 et 2004). La dispersion des mesures radiométriques sur une telle échelle demande encore à être expliquée (Fontugne, 2004). Par conséquent, la chronologie de la mise en place et de l'évolution des premiers Gravettiens en Europe occidentale et centrale s'avère d'autant plus délicate à appréhender.

Bien que la fléchette représente un lien indirect entre certains de ces Gravettiens (hormis les îles Britanniques et la Belgique), il n'existe pas d'ensemble véritablement identique au Bayacien de la Gravette. Les schémas, monocentristes ou polycentristes (Valoch, 1998) de diffusion du Gravettien depuis la Moravie et la Basse-Autriche reposent sur la présence de fléchettes dans le premier horizon gravettien de Willendorf (Otte, 1981, 1990 et 1991). La « route vers l'Ouest » de cette hypothèse diffusionniste s'appuie notamment sur le gisement d'Aggsbach $\mathrm{B}$, et de son rapprochement au Bayacien. Force est de constater que, en dépit de datations relativement récentes: GrN-1354: 25760 \pm 280 B.P.; GrN-2513: $26800 \pm 200$ B.P. (Otte, 1990), seule une mise en parallèle typologique d'outils assimilés à des fléchettes vient étayer cette hypothèse (Otte, 1981, 1985 et 1990). Cependant, le dernier niveau aurignacien de Willendorf (Teyssandier, 2003) ne peut en soit constituer un candidat valable à l'apparition du Gravettien localement. Les datations anciennes de Willendorf (Haesaerts et al., 1996) ont le mérite de proposer un calage chronologique à la mise en place du Gravettien sans étayer les processus conduisant à la structuration du Gravettien (Valoch, 1998; Otte, Noiret, 2004). «La question de l'origine du Gravettien n'est pas résolue »(Otte, Noiret, 2004, p. 25).

Les productions bayaciennes s'écartent profondément des normes en vigueur à la fin de l'Aurignacien. Les éléments notoires de chacun des systèmes, lamelles Dufour et fléchettes, relèvent d'intentions distinctes, voire contra- dictoires. Cette discontinuité se trouve à la base même du séquençage culturel. Or dans le cas présent, la comparaison des modalités techniques corrobore cette discontinuité. Mais si les processus diffèrent, la reconnaissance d'un changement profond dans la manière dont les Aurignaciens abordent la production lithique (Pesesse, Michel, 2006) ne permet pas non plus d'envisager à l'heure actuelle une solution de continuité entre ces groupes.

Aucune séquence ne documente précisément les modalités du passage de l'Aurignacien au Gravettien. Cette lacune archéologique amplifie les particularités de chaque technocomplexe, mais ce hiatus ne constitue en aucun cas la preuve d'une "rupture » entre ces deux entités culturelles. L'absence de lien n'a en effet jamais été démontrée et ne constitue qu'un héritage historiographique. Cette « rupture technique », en termes de processus et d'objectifs, s'explique par l'absence de jalons intermédiaires, mais cette apparente discontinuité ne traduit pas la nature des processus historiques sous-jacents. Cette « césure » pourrait être interprétée comme le fruit d'une accélération de l'évolution des systèmes techniques, en rapport ou non avec des mouvements de population. La rapidité de ce phénomène expliquerait alors en partie son faible enregistrement archéologique. D’autres sphères de la culture matérielle de ces populations pondèrent d'ailleurs cette impression de rupture, liée notamment à notre mode de compréhension des industries lithiques.

Le Bayacien, malgré sa position chronostratigraphique intermédiaire, ne peut donc être considéré comme une « industrie de transition ", dans le sens où il ne partage aucune convention commune avec l'Aurignacien. Dans cette perspective, le Bayacien correspond au premier enregistrement archéologique de l'émergence de nouvelles tendances dans la production lithique, foncièrement différentes des derniers savoir-faire aurignaciens et annonçant une nouvelle relation face au matériau et à l'équipement, au sein desquelles il est possible de reconnaître les normes gravettiennes à venir.

\section{Remerciements}

Mes remerciements s'adressent à C. Schwab, M.-S. Larguèze, S. Loizeau, ainsi que N. Rouquerol, L. Barbot et G. Odon pour leur accueil, à M. Lenoir pour m'avoir guidé dans la collection de Pair-non-Pair. Je remercie également J.-P. Bracco et L. Slimak pour leurs relectures. 


\section{BIBLIOGRAPHIE}

BOSSELIN B., DJINDJIAN F.

1994 : " La chronologie du Gravettien français ", Préhistoire européenne, 6, p. 7-115.

\section{BRÉZILLON M.}

1968 : La Dénomination des objets de pierre taillée: matériaux pour un vocabulaire des préhistoriens de langue française, Paris, CNRS Éditions (coll. Suppl. à Gallia Préhistoire, IV), 417 p., 227 pl.

\section{BRICKER H. M.}

1977 : « La contribution de l'abri Pataud à la question bayacienne ", Congrès préhistorique de France, $\mathrm{XX}^{e}$ session, Provence, 1-7 juillet 1974, Paris, Société préhistorique française, p. 48-52.

BRICKER H. M. (DIR.)

1995 : Le Paléolithique supérieur de l'abri Pataud (Dordogne) : les fouilles de H. L. Movius, Paris, Maison des sciences de l'homme (coll. Documents d'archéologie française, 50), $328 \mathrm{p}$

\section{Cheynier A., Daleau F.}

1963 : La Caverne de Pair-non-Pair (Gironde), Bordeaux, Publication de la Société archéologique de Bordeaux, Documents d'Aquitaine, $220 \mathrm{p}$.

\section{COMBIER J.}

1980 : « Circonscription Rhône-Alpes », Gallia Préhistoire, 23, p. 511-515.

1989 : « Aurignacien et Périgordien dans l'est de la France ", in MOHEN J.-P. (DIR.) Le Temps de la Préhistoire, XXIII Congrès préhistorique de France, Paris, Société préhistorique française et éd. Archéologia, vol. 1, p. 274-275.

\section{CONARD N. J., MOREAU L.}

2004 : "Current Research on the Gravettian of the Swabian Jura ", Mitteilungen der Gesellschaft für Urgeschichte, 13, p. 29-59.

\section{DELPORTE H.}

1972 : « L'Aurignacien et le Bayacien de la Gravette : mise en œuvre statistique et problèmes posés ", Bulletin de la Société préhistorique française, 69, ét. et trav. 1, p. $337-346$

\section{DEMARS P.-Y., LAURENT P.}

1989 : Types d'outils lithiques du Paléolithique supérieur en Europe, Paris, CNRS Éditions (coll. Cahiers du Quaternaire, 14).
DIGAN M.

2001 : Le Gisement gravettien de La Vigne-Brun (Loire) : première étude de l'industrie lithique de KL19, mémoire de Doctorat, Université Lille I, 2 vol.

\section{DJINDJIAN F., BosSELIN B.}

1994 : "Périgordien et Gravettien : l'épilogue d'une contradiction ? ", Préhistoire européenne, 6, p. 117-131.

ÉVIN J.

1982 : " Datations par radiocarbone du site de la Vigne Brun à Villerest (Loire) ", in COMbIeR J. (DIR.), Les Habitats $d u$ Paléolithique supérieur, Actes du colloque international en hommage au professeur André Leroi-Gourhan, Roanne-Villerest, 22-24 juin 1982, Lyon, DRAC Rhône-Alpes, 2 vol., p. $148-149$

\section{FONTUGNE M.}

2004 : «Les derniers progrès du calibrage des âges radiocarbones permettent-ils une révision des chronologies entre 25 et 50000 ans BP ? ", Quaternaire, 15, 3, p. $245-252$

\section{GENESTE J.-M.}

1982 : «Informations archéologiques de la circonscription d'Aquitaine : abri Vignaud", Gallia Préhistoire, 25, p. 415-416.

\section{GouTAS N.}

2004 : Caractérisation et évolution du Gravettien en France par l'approche techno-économique des industries en matières dures animales (étude de six gisements du Sud-Ouest), thèse de Doctorat, Université Paris-I-PanthéonSorbonne, $675 \mathrm{p}$.

\section{GUILLERMIN P.}

2004 : Réflexions sur linterprétation des industries gravettiennes à partir de l'étude techno-typologique d'une occupation spécialisée : la couche $\mathrm{E}$ du gisement des Fieux (Miers, Lot), mémoire de DEA, Université Toulouse-Le Mirail, $99 \mathrm{p}$.

haesaerts P., Borziak I., Chirica V., DAMBLON F., KOULAKOVSKA L.

2004 : "Cadre stratigraphique et chronologique du Gravettien en Europe centrale ", in Svoboda J., SEdlackova L. (DIR.), The Gravettian along the Danube, Proceedings of the Mikulov Conference, 20-21 nov. 2002, Brno, Institute of
Archeology, AS CR (coll. the Dolni Vestonice Studies, 11), p. 33-56.

\section{HAesaerts P., DAmblon F., BaChNer M.} TRNKA G.

1996 : « Revised Stratigraphy and Chronology of the Willendorf II Sequence, Lower Austria », Archeologia Austriaca, 80, p. $25-40$.

KLARIC L.

2003 : L'Unité technique des industries à burins $d u$ Raysse dans leur contexte diachronique : réflexions sur la diversité culturelle au Gravettien à partir des données de la Picardie, d'Arcy-sur-Cure, de Brassempouy et du Cirque de la Patrie, thèse de Doctorat, Paris, Université Paris-I-Panthéon-Sorbonne, $426 \mathrm{p}$.

2007 : «Regional Groups in the European Middle Gravettian : a Reconsideration of the Rayssian Technology », Antiquity, 81, p. 176-190.

\section{LACORRE $\mathbf{F}$.}

1933a : « Les armatures de flèches de la Gravette ", in $X V^{e}$ Congrès international d'anthropologie et d'archéologie préhistorique de Paris, 20-27 sept. 1931, Paris, Librairie E. Noury, p. 409-415.

1933b : " Les armatures de flèches de la Gravette : niveau et industries auxquelles elles se rattachent ", Procès-verbaux de la Société linéenne de Bordeaux, séance du 21 juin 1933, Bordeaux, Société linéenne, p. 78-87.

1960 : La Gravette, le Gravétien et le Bayacien, Laval, Imprimerie Barneoud.

\section{LE MENÉ F.}

1999 : Proposition pour une nouvelle approche de la pointe de la Font-Robert : les données de la Ferrassie et de Maisières-Canal, mémoire de Maîtrise, Université Paris-I

LEOZ L. E.

2001 : Contribution à l'étude du Gravettien de l'abri Pataud (Dordogne). Étude techno-typologique de lindustrie lithique du niveau 5 : Front, attribuée au Périgordien IV, mémoire de DEA, Muséum national d'histoire naturelle, Institut de Paléontologie humaine, $86 \mathrm{p}$.

MAZIÈre G., RAYNAL J.-P., DEMARS P.-Y., COURTY M.-A.

1984 : «Le gisement paléolithique supérieur de Puyjarrige 2 (Brive, Corrèze) ", Revue 
archéologique du Centre de la France, 23, 1, p. 21-67.

\section{NESPOULET $R$.}

1996 : Le Périgordien VI de l'abri Pataud, les Eyzies-de-Tayac, Dordogne : étude technologique et typologique de l'industrie lithique de la couche 3, thèse de Doctorat, Muséum national d'histoire naturelle.

\section{OTTE M.}

1981 : Le Gravettien en Europe centrale, Bruges, De Tempel (coll. Dissertationes archaeologicae gandenses, XX).

1985 : « Le Gravettien en Europe », L'Anthropologie, 89, p. 479-503.

1990 : «Révision de la séquence du Paléolithique supérieur de Willendorf (Autriche) ", Bulletin de l'Institut royal des sciences naturelles de Belgique, 60, p. 219-228.

1991 : «Révision de la séquence de Willendorf ", in MONTET-WHite A. (DIR.), Les Bassins du Rhin et du Danube au Paléolithique supérieur : environnement, habitat et systèmes d'échange, Colloque de la commission VIII "Paléolithique supérieur", Actes du XI congrès de l'Union internationale des sciences préhistoriques et protohistoriques, Mayence, 1986, Liège, Service de Préhistoire (coll. ERAUL, 43), p. 45-60.

\section{OTTE M., NOIRET P.}

2004 : «Évolution du Gravettien au moyen Danube », in SVOBODA J., SEDLACKOVA L. (DIR.), The Gravettian along the Danube, Proceedings of the Mikulov Conference, 20-21 nov. 2002, Brno, Institute of Archeology, AS CR (coll. the Dolni Vestonice Studies, 11), p. 8-32.

\section{PELEGRIN J.}

2000 : « Les techniques de débitage laminaire au Tardiglaciaire : critères de diagnose et quelques réflexions ", in VAlentin B., BODU P., ChRISTEnSEN M. (DIR.), L'Europe centrale et septentrionale au Tardiglaciaire, Actes de la table ronde de Nemours, 14-16 mai 1997, Nemours, APRAIF, (coll. Mémoires du musée de Préhistoire d'Île-de-France, 7), p. 73-86.

\section{PERPÈRE M.}

1992 : «Contribution à l'étude des pointes de traits périgordiennes : les fléchettes ", Préhistoire européenne, 2, p. 89-95.

\section{PESESSE D.}

2003 : Approche du comportement technique au Gravettien : l'industrie lithique de l'unité OP10 de la Vigne Brun, mémoire de DEA, Université Aix-Marseille-I.

2006: «La "pointe à dos alternes" : un nouveau fossile directeur du Gravettien ? ", Bulletin de la Société préhistorique française, 103, 3, p. $465-478$.

\section{PeSESSE D., Michel A.}

2006 : «Le burin des Vachons : apports d'une relecture technologique à la compréhension de l'Aurignacien récent du nord de l'Aquitaine et des Charentes ", Paléo, 18, p. 143-159.

\section{PEYRONY D.}

1933 : «Les industries aurignaciennes dans le bassin de la Vézère ", Bulletin de la Société préhistorique française, 30, p. 543-559.

1943 : «Le gisement du Roc de CombeCapelle, commune de Saint-Avit-Sénieur (Dordogne) ", Bulletin de la Société historique et archéologique en Périgord, 70, p. 158-173.

1947 : «Le remplissage des vallées de la Vézère et de la Beûne ", Gallia, 5, p. 180-184.

\section{POtTier C.}

2005 : Le Gravettien moyen de l'abri Pataud (Dordogne, France), le niveau 4 et l'éboulis $3 / 4$ : étude technologique et typologique de l'industrie lithique, thèse de Doctorat, Paris, Muséum national d'histoire naturelle, Institut de Paléontologie humaine, 396 p.

\section{RIGAUD J.-P.}

1998 : «L'origine du Gravettien dans le sud de la France ", in PALMA DI CESNOLA A., VALOCH K. (DIR.), XIII international Congress of Prehistoric and Protohistoric Sciences, Forli, 8-14 sept. 1996, Colloqium XII : The Origin of the Gravettian, Forli, ABACO edizioni, p. 257-262.

\section{SIMONET A.}

2004 : L'Atelier de taille gravettien de Tercis (Landes). Approche technologique, économique et sociologique. Étude de l'industrie lithique de deux concentrations dites " à pièces à dos " et "à grandes lames ", mémoire de Maîtrise, Université Toulouse-II-Le Mirail.

2005 : Les Armatures lithiques. Méthodes d'étude et enjeux : l'exemple des armatures gravettiennes d'Isturitz, mémoire de DEA, Université Toulouse-II-Le Mirail.

\section{SONNEVILLE-BORDES D.}

1960 : Le Paléolithique supérieur en Périgord, Bordeaux, Delmas, 2 vol., 558 p.

Surmely F., Alix P., Costamagno S., DANIEL P., HAYS M., MURAT R., RENARD R., VIRMONT J., TEXIER J.-P.

2003 : «Découverte d'un gisement gravettien ancien au lieu-dit le Sire (Mirefleurs, Puy-de-Dôme) », Bulletin de la Société préhistorique française, 100, 1, p. 29-40.

\section{TEYSSANDIER N.}

2003 : Les Débuts de l'Aurignacien en Europe : discussion à partir des sites de Geissenklösterle, Willendorf II, Krems-Hundssteig et Bacho Kiro, thèse de Doctorat, Université Paris$\mathrm{X}$-Nanterre.

\section{VALOCH K.}

1998 : «L'origine du Gravettien de l'Europe centrale ", in PALMA DI CESNOLA A., VALOCH K. (DIR.), XIII international Congress of Prehistoric and Protohistoric Sciences, Forli, 8-14 sept. 1996, Colloqium $X I I$ : " the Origin of the Gravettian ", Forli, ABACO edizioni, p. 203-212. 